\title{
M1- and M2-Type Macrophage Responses Are Predictive of Adverse Outcomes in Human Atherosclerosis
}

\author{
Monica de Gaetano ${ }^{1}$, Daniel Crean ${ }^{2}$, Mary Barry ${ }^{3}$ and Orina Belton ${ }^{1 *}$ \\ ${ }^{1}$ School of Biomolecular and Biomedical Science, UCD Conway Institute, University College Dublin, Dublin, Ireland, ${ }^{2}$ School \\ of Veterinary Medicine, UCD Conway Institute, University College Dublin, Dublin, Ireland, ${ }^{3}$ St. Vincent's University Hospital, \\ Dublin, Ireland
}

OPEN ACCESS

Edited by:

Claudia Monaco,

University of Oxford, UK

Reviewed by:

Angelo A. Manfredi,

Vita-Salute San Raffaele

University, Italy

Charles Dudley Mills,

BioMedical Consultants, USA

*Correspondence:

Orina Belton

orina.belton@ucd.ie

Specialty section: This article was submitted to Inflammation,

a section of the journal

Frontiers in Immunology

Received: 08 March 2016 Accepted: 01 July 2016

Published: 19 July 2016

Citation:

de Gaetano M, Crean D, Barry M and Belton O (2016) M1- and

M2-Type Macrophage Responses Are Predictive of Adverse Outcomes in Human Atherosclerosis. Front. Immunol. 7:275.

doi: 10.3389/fimmu.2016.00275
Atherosclerosis is an inflammatory disease caused by endothelial injury, lipid deposition, and oxidative stress. This progressive disease can be converted into an acute clinical event by plaque rupture and thrombosis. In the context of atherosclerosis, the underlying cause of myocardial infarction and stroke, macrophages uniquely possess a dual functionality, regulating lipid accumulation and metabolism and sustaining the chronic inflammatory response, two of the most well-documented pathways associated with the pathogenesis of the disease. Macrophages are heterogeneous cell populations and it is hypothesized that, during the pathogenesis of atherosclerosis, macrophages in the developing plaque can switch from a pro-inflammatory $(M \Phi 1)$ to an anti-inflammatory (MФ2) phenotype and vice versa, depending on the microenvironment. The aim of this study was to identify changes in macrophage subpopulations in the progression of human atherosclerotic disease. Established atherosclerotic plaques from symptomatic and asymptomatic patients with existing coronary artery disease undergoing carotid endarterectomy were recruited to the study. Comprehensive histological and immunohistochemical analyses were performed to quantify the cellular content and macrophage subsets of atherosclerotic lesion. In parallel, expression of $M \Phi 1$ and $M \Phi 2$ macrophage markers were analyzed by real-time PCR and Western blot analysis. Gross analysis and histological staining demonstrated that symptomatic plaques presented greater hemorrhagic activity and the internal carotid was the most diseased segment, based on the predominant prevalence of fibrotic and necrotic tissue, calcifications, and hemorrhagic events. Immunohistochemical analysis showed that both $\mathrm{M} \Phi 1$ and $\mathrm{M} \Phi 2$ macrophages are present in human plaques. However, MФ2 macrophages are localized to more stable locations within the lesion. Importantly, gene and protein expression analysis of M $\Phi 1$ /

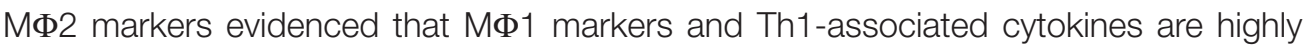
expressed in symptomatic plaques, whereas expression of the MФ2 markers, mannose receptor (MR), and CD163 and Th2 cytokines are inversely related with disease progression. These data increase the understanding of atherosclerosis development, identifying the cellular content of lesions during disease progression, and characterizing macrophage subpopulation within human atherosclerotic plaques.

Keywords: atherosclerosis, inflammation, macrophage phenotype, human plaque 


\section{INTRODUCTION}

Atherosclerosis is a progressive disease characterized by the accumulation of lipids and fibrous elements in large- and mediumsized arteries, driven by several genetic and environmental factors (1-4). A large number of risk factors for the disease have been identified, such as elevated low-density lipoprotein (LDL) cholesterol, hypertension, obesity, and type- 2 diabetes. Atherosclerotic plaques are initiated by the accumulation of LDL and the recruitment of inflammatory cells in particular monocytes, at sites of endothelial dysfunction (5). Monocyte migration and their differentiation into macrophages by exposure to macrophage colony-stimulating factor (M-CSF) is followed by subsequent foam cell formation, arising from macrophage receptor-mediated uptake of oxidized LDL (ox-LDL), and a proliferative response of smooth muscle cells (6). Plaque rupture triggers $\sim 76 \%$ of all fatal coronary thrombi (7). Established atherosclerotic patients are classified as either "symptomatic," where the patient has experienced previous ischemic events but without any cardiovascular disease (CVD) diagnosis; or "asymptomatic," where a patient has no history of ischemic events or CVD. Among patients with symptomatic carotid artery stenosis, surgical removal of the advanced plaque, by carotid endarterectomy (CEA), is still one of the most performed surgical procedures, due to its associated lower risk of peri-procedural stroke or death, in comparison with other types of intervention, such as carotid artery stenting (8). Although conventional therapies, such as statins, which decrease plasma LDL levels, and antihypertensives control the risk factors for the disease, there are currently no available therapies that effectively regress pre-established plaques (9). The implications of this are important as most people present clinically with established disease and the therapeutic goal would be to reverse the established lesion. While most of the cellular and molecular mechanisms in the development of atherosclerotic lesions have been identified, there is limited information on those pathways and processes governing regression. Indeed, identifying novel cellular processes associated with atheroprotection or those which regulate regression is critical to the identification of novel therapeutic targets.

Macrophages are phagocytic cells derived from bone marrow precursors and monocytes. They are essential for the maintenance and defense of host tissues, as they sense and engulf cellular debris and pathogens. They are also able to stimulate a pro-inflammatory response by stimulating lymphocytes and other immune cells to respond to the pathogen (10). One of the most critical functions of macrophages, in the context of atherosclerosis, is lipid uptake and deposition and the subsequent disease progression. ox-LDL is recognized by macrophage scavenger receptors (SRs), predominantly CD36 and CD68. SRs mediate the uptake of highly ox-LDL by macrophages, leading to an increase in intracellular lipid accumulation and consequently, foam cell formation (11). Intracellular cholesterol is then metabolized and transported to exogenous acceptors, such as high-density lipoprotein, through efflux proteins, such as ATP-binding cassette $(\mathrm{ABC})$ transporters, ABCA1 and ABCG1 (12-14).

We have previously shown that dietary administration of conjugated linoleic acid (CLA), a family of naturally occurring geometric dienoic isomers of the $\omega 6$ essential fatty acid, linoleic acid (15) induces regression of pre-established atherosclerosis in the apoE mouse model of atherosclerosis despite a continuing high cholesterol challenge (16). Furthermore, we have recently identified the monocyte/macrophage as the cellular target through which CLA mediates this profound effect $(17,18)$. Of relevance to this present study, we have shown, in both in vivo and in vitro, that CLA primes macrophages toward an anti-inflammatory macrophage phenotype and limits macrophage accumulation and foam cell formation by modulating the expression of both SRs and efflux proteins, ultimately, altering cholesterol trafficking in regression of pre-established atherosclerosis $(19,20)$. This suggests that altering the macrophage phenotype may be of therapeutic importance in established disease. However, currently there is limited information regarding the localization and function of macrophage subpopulations in the progression of atherosclerosis particularly in progression from asymptomatic to symptomatic disease.

Over the last decade, the heterogeneity of macrophage populations has been extensively documented (21). Two types of macrophages have been identified based on the exposure to different cytokine environments. The "classically activated," $M \Phi 1$, pro-atherogenic macrophages are primed by exposure to $T$ helper (Th)-1 cytokines, such as IFN $\gamma$ and IL- $1 \beta$; while "alternative," $\mathrm{M} \Phi 2$, anti-inflammatory macrophages are induced as a result of exposure to Th2 cytokines, such as IL-4 and IL-3 (22). Macrophages are phenotypically plastic cells, in that they can switch from MФ1 to $M \Phi 2$ state and vice versa upon specific signals $(23,24)$. Due to their enhanced phagocytic activity in early plaque development, the anti-inflammatory properties of MФ2 macrophages, in comparison to $M \Phi 1$ pro-inflammatory, macrophages have been highlighted (24). It is suggested that atherosclerosis may be caused not only by a sustained pro-inflammatory reaction but also by impaired anti-inflammatory, pro-resolving mechanisms (21). For example, it has been shown that the presence of macrophages in human atherosclerotic plaques are indicative of a chronic inflammatory reaction (25). The majority of previous studies identifying macrophage subsets in human atherosclerosis have compared atherosclerotic plaques with normal vessels and while they have confirmed the heterogeneity of the macrophage subpopulations (26), data on macrophage plasticity in the context of human atherosclerotic disease progression is limited. Shaikh et al. showed using immunohistochemistry analysis, that plaques from patients with recently symptomatic carotid disease have a predominance of M1 macrophages and higher lipid content compared to femoral plaques, consistent with a more unstable plaque (27). Of relevance to this study is that the relationship between macrophage polarization and the vulnerability of human atherosclerotic plaques has been investigated. Macrophage-rich areas were identified by $\mathrm{CD}^{+} 8^{+}$immunostaining and showed that plaques from symptomatic patients had a greater concentration of macrophages specifically M1 macrophages. By contrast, increased expression of markers associated with alternative M2 macrophage differentiation was observed in plaques from the asymptomatic group (28).

The focus of this study was to comprehensively analyze macrophage subsets in human atherosclerosis progression by 
comparing cellular content and macrophage populations in plaques from asymptomatic and symptomatic patients. To this end, several macrophage markers were analyzed. Expression of the ox-LDL SRs, CD36 and CD68 was examined, as although both receptors mediate uptake of modified lipoprotein, they have different cellular localizations. CD36 is expressed on the plasma membrane and has previously been used to identify both MФ1 and MФ2 macrophage subsets, while CD68 is located on the lysosomal surface $(25,29)$. To examine the expression of cytokines known to induce an $M \Phi 1$ phenotype, we performed mRNA expression analysis of Th1-cytokines (TNF $\alpha$, IL-1 $\beta$, IL-6, IL-8, IL-12p40, and IL-12p35) and of Th1-chemokines (MCP-1 and CXCL10) (30). In addition, MR, CD163 and Dectin-1 were used as established MФ2 markers $(24,27,31,32)$. MR is a type-I membrane receptor protein that is found on the macrophage surface, where it mediates the endocytosis of glycoproteins by binding high-mannose structures on the surface of potentially pathogenic viruses, fungi, and bacteria, enabling them to be neutralized by phagocytic engulfment. During inflammation, $\mathrm{MR}$ is crucial for rapid clearance of several mannose-bearing serum glycoproteins but does not regulate the initiation of inflammation $(33,34)$. The hemoglobin-heptaglobin SR, also known as CD163, is a macrophage-specific protein and a hallmark of the macrophage switch to MФ2 phenotype (35). Dectin- 1 is a $\beta$-glucan receptor expressed on leukocytes, mediating all the immunomodulatory effects of carbohydrates, including the $\beta$-glucan-dependent binding of zymosan, a yeastderived particle composed principally of polysaccharides (32). Moreover, Dectin-1 has been recently associated with the production of high levels of IL-10 but low levels of IL-12p40, thus, shifting the macrophage phenotype toward an $\mathrm{M} 2 \mathrm{~b}$ or "regulatory" macrophage (36). Furthermore, we also examined expression of cytokines (IL-10, IL-4, and IL-13) and chemokines (CCL22 and CCL18) which are known to induce an MФ2 phenotype (37).

There remains a lack of consensus on whether the cholesterol efflux proteins, ABCA1 and ABCG1, and the SR, SRA-1, are associated with an MФ1 or MФ2 phenotype $(24,38)$. ABCA1 exports cholesterol to lipid-free apolipoproteins (apo), involving an initial interaction of apoAI with lipid rafts located on the macrophage surface, while ABCG1 effluxes cholesterol to phospholipidcontaining acceptors, such as HDL, in a lipid raft-independent manner $(27,39)$. The canonical route for ox-LDL to enter the cell is via SR-mediated uptake and it has been extensively shown that the pro-inflammatory cytokine, IFN- $\gamma$, increases the expression of the SR SRA-1, in both THP-1- and HBPMC-derived macrophages (40).

Thus, the expression of ABCA1, ABCG1, and SRA-1 in asymptomatic and symptomatic atherosclerotic plaques was also examined to identify if they are altered in disease progression and to establish if they are predominantly associated with an $M \Phi 1$ or МФ2 phenotype.

Therefore, the overall aim of the present study was to elucidate the differential macrophage populations in atherosclerosis progression, through the analysis of cellular content and the relative distribution of $M \Phi 1$ and $M \Phi 2$ macrophage populations in human asymptomatic and symptomatic plaques.

\section{MATERIALS AND METHODS}

\section{Patients}

\section{Tissue Specimen Preparation}

The study was approved by the Ethics Committee of St. Vincent's University Hospital, Dublin and in accordance with International guidelines and Helsinki Declaration principles. All patients gave written informed consent. Patients with clinical and angiographic evidence of atherosclerosis undergoing revascularization surgery were recruited to the study. Immediately following CEA, human carotid atherosclerotic plaques specimens were either fixed in formalin for $24 \mathrm{~h}$, and stored at room temperature for subsequent immunohistochemical analysis, or placed in "RNA later" solution, and stored at $-80^{\circ} \mathrm{C}$ for RNA and protein analysis. Photographs were taken of whole plaques prior to dissection, in order to document the dorsal and ventral orientation of the specimen. Each plaque specimen was then dissected into four distinct sections: common carotid (CC), internal carotid (IC), external carotid (EC), and relatively disease free (RDF), as shown in Figure 1A. Each plaque section was individually placed in plastic cassettes and processed for $9 \mathrm{~h}$ through a cycle of alcoholic and organic solvents, ending with a wax-embedding step, using an automated instrument, before being manually embedded in paraffin wax, as described in detail in Table S1 in Supplementary Material.

Paraffin-embedded sections were then cut into slices of $6 \mu \mathrm{m}$ of thickness. This process was used to obtain serial sections of each sample to be analyzed. The paraffin sections were mounted on polylysine-coated slides in a water bath at $37^{\circ} \mathrm{C}$. The slides were then placed in the histology oven for $1 \mathrm{~h}$ to secure adhesion of tissue to the slide.

\section{H\&E Staining}

After deparaffinization in xylene and subsequent rehydration in graded alcohol series (Table S2 in Supplementary Material), Mayer's Hematoxylin (HX) (Sigma Aldrich, Ireland) was applied to each tissue section for $5 \mathrm{~min}$, in order to stain nuclei. After washing, acidic ethanol $(99 \mathrm{ml}$ of $70 \%$ ethanol and $1 \mathrm{ml}$ of Hydrochloric acid) was added for $5 \mathrm{~s}$ to de-stain. Eosin (Merck, Ireland) was then added to each section for $5 \mathrm{~min}$ to visualize the cytoplasm and the extracellular matrix of the tissue. Slides were then washed and dehydrated as described in Table S3 in Supplementary Material. Mounted slides were allowed to dry overnight before transmission light microscopy (TLM) visualization, using Nikon Eclipse $80 \mathrm{i}$ and the NIS-Elements BR software.

\section{IHC Staining Procedure}

After deparaffinization in xylene and subsequent rehydration in graded alcohol series, endogenous peroxidases were quenched using $3 \% \mathrm{H}_{2} \mathrm{O}_{2}$ (Merck, Ireland) in 30\% methanol (Sigma Aldrich, Dublin, Ireland) for $30 \mathrm{~min}$. After washing, non-specific antibody binding was prevented by blocking with serum in which the antibody was raised for $1 \mathrm{~h}$ at room temperature. Following washes, antibodies against human CD68 (1:100), SMA (1:100) (both from DAKO, Glostrup Denmark) and MR (1:50) (Sigma Aldrich, Dublin, Ireland) were incubated overnight at $4^{\circ} \mathrm{C}$. After washing, secondary biotinylated 


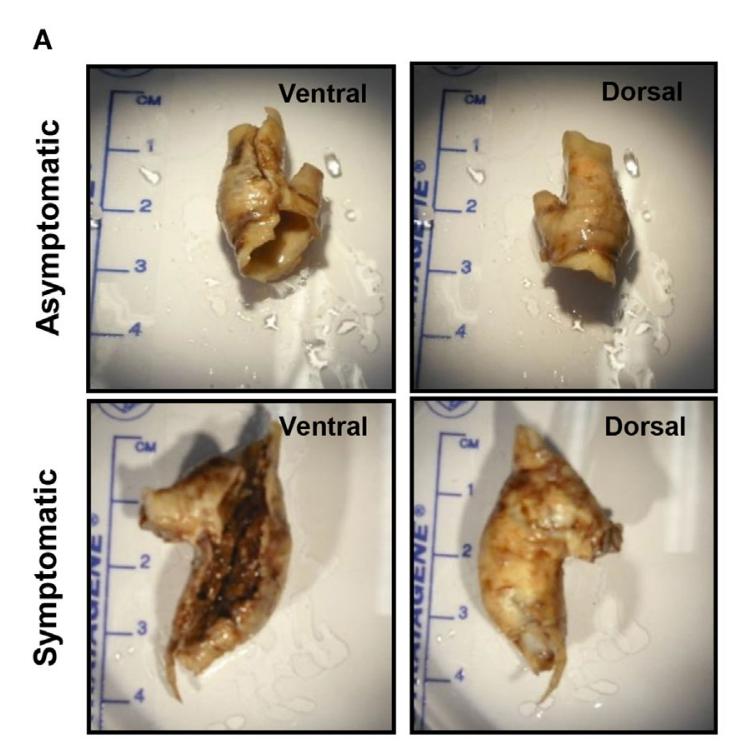

B

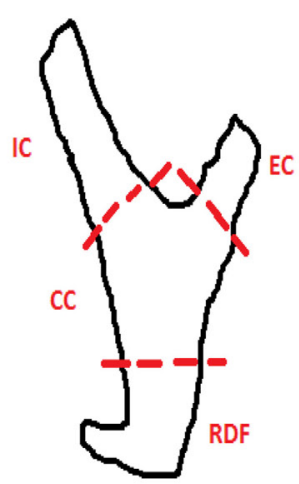

C

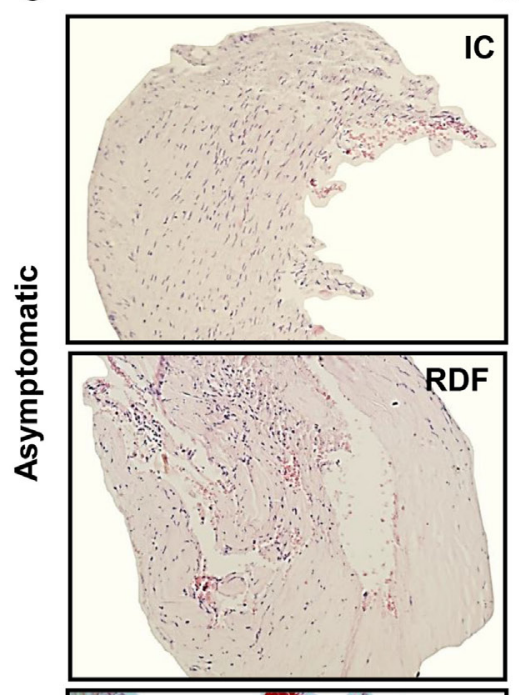

D

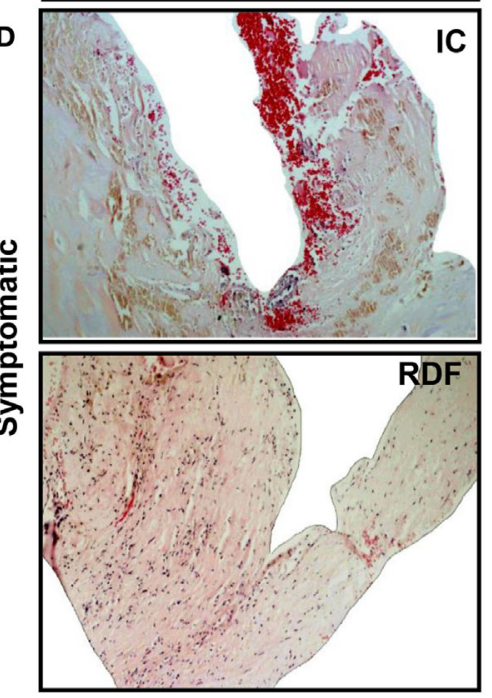

$10 \mathrm{x}$
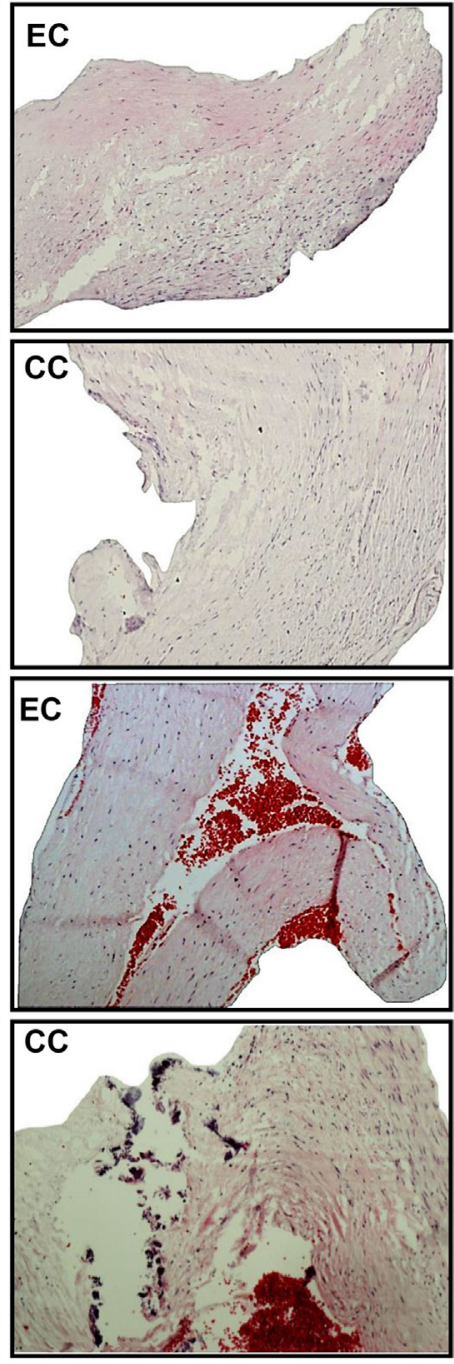

FIGURE 1 | Comprehensive analysis of asymptomatic vs. symptomatic plaque. (A) Gross analysis. After collection, atherosclerotic plaque tissues were analyzed at gross level in order to detect evident signs of hemorrhage or calcification and to measure plaque size. Generally, asymptomatic patients presented smaller lesions with less evident signs of hemorrhage or calcification, compared to symptomatic patients. Here, representative images for each group of patients are displayed. (B) Dissection. Schematic of carotid atherosclerotic plaque dissection where dotted lines indicate the points of dissection, and the resulting sections are labeled as follows: common carotid (CC), the main arterial element and origin of disease; internal carotid (IC), the larger bifurcation from the CC; external carotid $(E C)$, the smaller bifurcation from the $C C$ and the relatively disease-free (RDF) component, least diseased region of the artery due to its peripheral location. (C,D) H\&E staining. After dissection, H\&E staining was performed on $6 \mu \mathrm{m}$ sections. A representative comparison between (C) asymptomatic and (D) symptomatic plaque is shown. Hemorrhagic signs, calcification, and necrotic depositions are clearly visible in all dissected sections from the symptomatic plaques, with the exception of RDF. Pictures were captured using TLM (10x magnification). Representative images of $N=3$ independent staining are displayed here.

horseradish peroxidase (HRP) Ab was incubated for $1 \mathrm{~h}$ at room temperature. Once washed, VECTASTAIN Universal Elite ABC solution (Vector Laboratories), an Avidin Biotinylated peroxidase Complex, was added for $30 \mathrm{~min}$. Following washing steps, 3,3'-Diaminobenzidine (DAB, Sigma Aldrich, Dublin, Ireland) brownie chromogen was placed for $2-5 \mathrm{~min}$ as peroxidase substrate to localize peroxidase in tissue. Mayer's HX was used as counterstain for $90 \mathrm{~s}$. After washing, dehydration steps followed. Mounted slides were allowed to dry before TLM visualization or before scanning with Aperio ScanScope XT digital scanner. Negative controls were obtained omitting addition of primary $\mathrm{Ab}$, secondary $\mathrm{Ab}, \mathrm{DAB}$ or $\mathrm{HX}$, as shown in Figure S1 in Supplementary Material. All subsequent analysis and quantification was performed by a trained researcher blinded to the symptomatic status of the patients.

\section{IHC Staining Visualization and Quantification}

Stained slides were visualized using the Aperio ScanScope XT digital scanner and its software Aperio ScanScope Console. Images were then stored on the "Spectrum" platform for analysis. 
IHC staining was quantified using the Aperio "ImageScope" software and its required algorithms. The first algorithm used was "Colour Deconvolution," which calibrated the immunostaining for $\mathrm{DAB}$ and $\mathrm{HX}$ of each plaque sample stained, giving a basal level of the target protein expression.

Nuclear analysis was then carried out to, first, establish the target protein expression per cell and, second, as a total per tissue section. "V9 Nuclear algorithm," using the baseline values previously produced by Colour Deconvolution, quantified the intensity of DAB staining (protein) in each HX (nuclei) stained cell and assigned a mark-up image that was more easily quantifiable. The mark-up images applied different arbitrary colors to corresponding levels of protein expression. Counting of cells, for each different color-coded level of protein expression, was elaborated by the software, as we have previously described (41).

For each tissue section, every 10 slides cut (composing a "series" progressively numbered), one slide was stained using the same primary antibody, in order to have three "series" of slides (60 $\mu \mathrm{m}$ apart) throughout the tissue; thus, representative of the whole specimen analyzed (for example, CD68 staining was performed on the 1st, 11 th, and 21 st slide; MR on the $2 \mathrm{nd}, 12 \mathrm{th}$, and 22th; SMA on the 3rd, 13th, and 23rd). An average quantification of the three series for each marker was then calculated.

\section{Gene Expression Analysis}

For gene expression experiments, specimens stabilized in RNA later solution were used to extract total RNA from tissues using Trizol (Invitrogen, Ireland). Reverse transcription was carried out on $1 \mu \mathrm{g}$ of total RNA using Superscript ${ }^{\mathrm{Tm}}$ III Reverse Transcriptase (Invitrogen, Ireland) according to the manufacturers' instructions. Relative gene expression quantification by real-time PCR (RT-PCR) was performed on an ABI Prism 7900HT Sequence Detection System (Applied Biosystem Inc., UK). MR, SRA-1, and ABCG1 expression were examined using specific Taqman assays (Applied Biosystems Inc., UK), while TNF $\alpha$, IL-1 $\beta$, IL-6, IL-8, IL-12p40, IL-12p35, CXCL10, MCP-1, Dectin-1, CCL22, CCL18, IL-10, IL-4, IL-13, ABCA1, CD14, CD36, CD68, and CD163 target genes were measured using specific Syber green assays (Eurofins, MWG Operon, Germany) and $\mathrm{Ct}$ values were normalized to $18 \mathrm{~s}$ ribosomal RNA (Table S4 in Supplementary Material).

\section{Western Blot Analysis}

Total tissue protein lysates were separated using 4-20\% SDSpolyacrylamide gel and transferred to nitrocellulose membranes. Membranes were blocked with 3\% BSA in TBS-T at room temperature prior to overnight incubation with primary antibodies at $4^{\circ} \mathrm{C}$ with gentle shaking. Detection was performed using a HRP-conjugated secondary antibody incubated for $1 \mathrm{~h}$ at room temperature and ECL detection system. Mouse-monoclonal anti-human $\beta$-actin (1:1000); goat-polyclonal anti-human CD68 (1:500); rabbit-polyclonal anti-human MR (1:500); and ABCA1 (1:1000) were used. $\beta$-actin, CD68, and MR were purchased from Santa Cruz Biotechnology, CA, USA. ABCA1 was purchased from Novus, CO, USA. The following HRPconjugated secondary antibodies were as follows: polyclonal anti-rabbit secondary antibody (Cell Signaling Technology, MA,
USA); polyclonal anti-mouse secondary antibody (Santa Cruz Biotechnology, CA, USA).

\section{Statistical Analysis}

The data were analyzed by parametric unpaired Student's $t$-test and analysis of variance (ANOVA). The student $t$-test was used to compare the mean of two data sets. ANOVA was used to examine any overall differences between groups. Results are expressed as mean \pm SEM. Experimental points were performed in triplicate with a minimum of three independent experiments. A value of $p<0.05$ or smaller was considered statistically significant.

\section{RESULTS}

\section{Patients}

Eighty-seven patients undergoing CEA were recruited to this study, three of which had bilateral lesions. The "symptomatic status" of the patients at the time of surgery was available for 80 patients based on their clinical records. Thirty-four out of 80 patients (42.5\%) were classified as "symptomatic," while 46 out of

\begin{tabular}{lcccc}
\hline TABLE 1 | Population profile. & & & \\
\hline & $\begin{array}{c}\text { CEA } \\
\text { frequency } \\
\text { relative to } \\
\text { gender, } \boldsymbol{N} \text { (\%) }\end{array}$ & $\begin{array}{c}\text { CEA frequency } \\
\text { relative to } \\
\text { symptomatic } \\
\text { status, } \boldsymbol{N} \text { (\%) }\end{array}$ & $\begin{array}{c}\text { Average } \\
\text { age }\end{array}$ & Range \\
& $80(100)$ & $80(100)$ & 68.0 & $47-83$ \\
All patients & $34(42.4)$ & $34(100)$ & 70.2 & $51-83$ \\
Symptomatic & $46(57.5)$ & $46(100)$ & 65.6 & $47-83$ \\
Asymptomatic & $60(100)$ & $60(75)$ & 67.1 & $47-83$ \\
Males & $24(40)$ & $24(71)$ & 67.9 & $51-83$ \\
Symptomatic & $36(60)$ & $36(78)$ & 65.8 & $47-83$ \\
Asymptomatic & $20(100)$ & $20(15)$ & 69.9 & $60-83$ \\
Females & $10(50)$ & $10(29)$ & 74.6 & $65-83$ \\
Symptomatic & $10(50)$ & $10(22)$ & 65.2 & $60-70$ \\
Asymptomatic & & & & \\
\hline
\end{tabular}

Characteristics (gender, age, and symptomatic status) of the 80 patients recruited to the study that underwent CEA. The average age is higher in symptomatic vs. asymptomatic, in female vs. male and in symptomatic females vs. symptomatic males.

TABLE 2 | Characteristics of patients analyzed in the study.

\begin{tabular}{lcccr}
\hline & $\begin{array}{c}\text { CEA } \\
\text { frequency } \\
\text { relative to } \\
\text { gender, } \boldsymbol{N} \text { (\%) }\end{array}$ & $\begin{array}{c}\text { CEA frequency } \\
\text { relative to } \\
\text { symptomatic } \\
\text { status, } \boldsymbol{N} \text { (\%) }\end{array}$ & $\begin{array}{c}\text { Average } \\
\text { age }\end{array}$ & Range \\
\hline All patients & $18(100)$ & $18(100)$ & 64.6 & $41-83$ \\
Symptomatic & $9(50)$ & $9(100)$ & 70.4 & $41-83$ \\
Asymptomatic & $9(50)$ & $9(100)$ & 58.7 & $43-71$ \\
Males & $15(100)$ & $15(83)$ & 63.0 & $41-83$ \\
Symptomatic & $7(47)$ & $7(78)$ & 68.1 & $41-83$ \\
Asymptomatic & $8(53)$ & $8(89)$ & 58.5 & $43-71$ \\
Females & $3(100)$ & $3(17)$ & 72.6 & $61-79$ \\
Symptomatic & $2(67)$ & $2(22)$ & 78.5 & $78-79$ \\
Asymptomatic & $1(37)$ & $1(11)$ & 61 & -
\end{tabular}

Plaques from 18 patients that were analyzed for either H\&E/IHC analysis or for RNA/protein expression. Table shows gender, age, and symptomatic status. The average age is higher in symptomatic vs. asymptomatic, in female vs. males and in symptomatic females vs. symptomatic male. The plaques from the patients analyzed in this study are representative of the whole population recruited as described in Table 1. 
$80(57.5 \%)$ were defined as "asymptomatic." The average age of all patients was $68 \pm 3.6$. However, the average age was slightly higher in the symptomatic $(71 \pm 7.8$ years) group than in the asymptomatic group $(66 \pm 7.6$ years) (Table 1$)$. The characteristics of the patients analyzed in this study for IHC or mRNA/ protein analyzes displayed a similar trend as the one described above (Table 2).

\section{Atherosclerotic Plaque Gross Analysis}

Prior to dissection of the plaque samples, the dorsal and ventral surface of each specimen was photographed to detect gross calcification and identify evidence of hemorrhagic plaques. Figure 1A shows representative plaques from symptomatic and asymptomatic patients. All plaques analyzed from asymptomatic patients displayed no evidence of calcification and very little or no hemorrhagic spots. By contrast, all symptomatic plaques had extensive hemorrhaging and had partial or severe calcification. The average size of asymptomatic plaques was smaller than that of symptomatic $(2.8 \pm 0.4$ vs. $3.8 \pm 0.2 \mathrm{~cm})$, which is in keeping with the assumption that symptomatic patients present with more advanced and/or complicated lesions. Therefore, gross analysis of plaque specimens confirmed that comparison of asymptomatic and symptomatic plaques is a valid model of disease progression.

\section{H\&E Analysis of Asymptomatic and Symptomatic Plaques}

H\&E staining was performed on sections from all plaques. Figure 1 shows a representative overview of the cellular composition of plaques from both asymptomatic and symptomatic patients. Dotted lines in the diagram indicate how the surgically removed plaque specimen was dissected into "CC," the area where vessel bifurcation originates, which is also the most prone region for plaque development, "IC," the main arterial element and site of origin of disease, at the interphase with CC; "EC," the smaller bifurcation from the CC and, finally, the "RDF" component, least diseased region, due to its peripheral location that was used as a control (Figure 1B). Asymptomatic plaques were homogeneous in terms of cellular content and absence of hemorrhagic signs (platelets and red blood cells stained in red), except for evidence of early hemorrhagic activity in the IC and EC sections, which are located at the site of bifurcation of the vessel, representing the more atheroprone regions (Figure 1C). Conversely, plaques from symptomatic
A
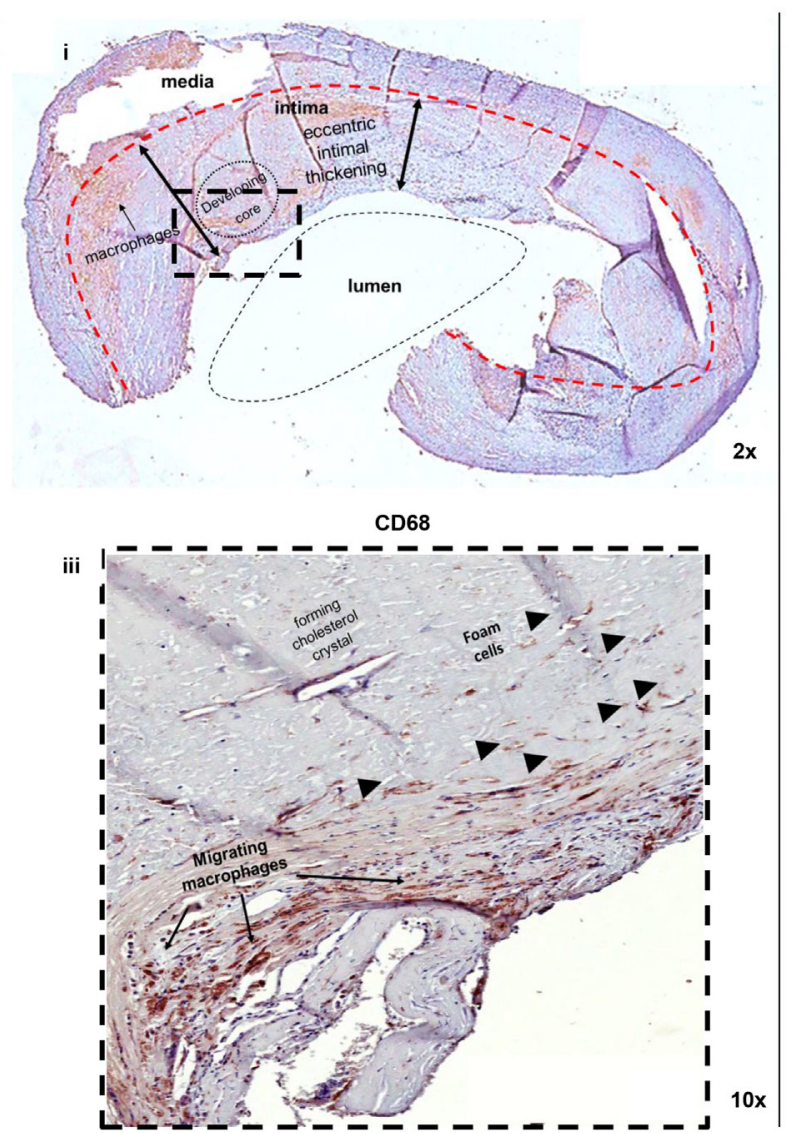

Asymptomatic

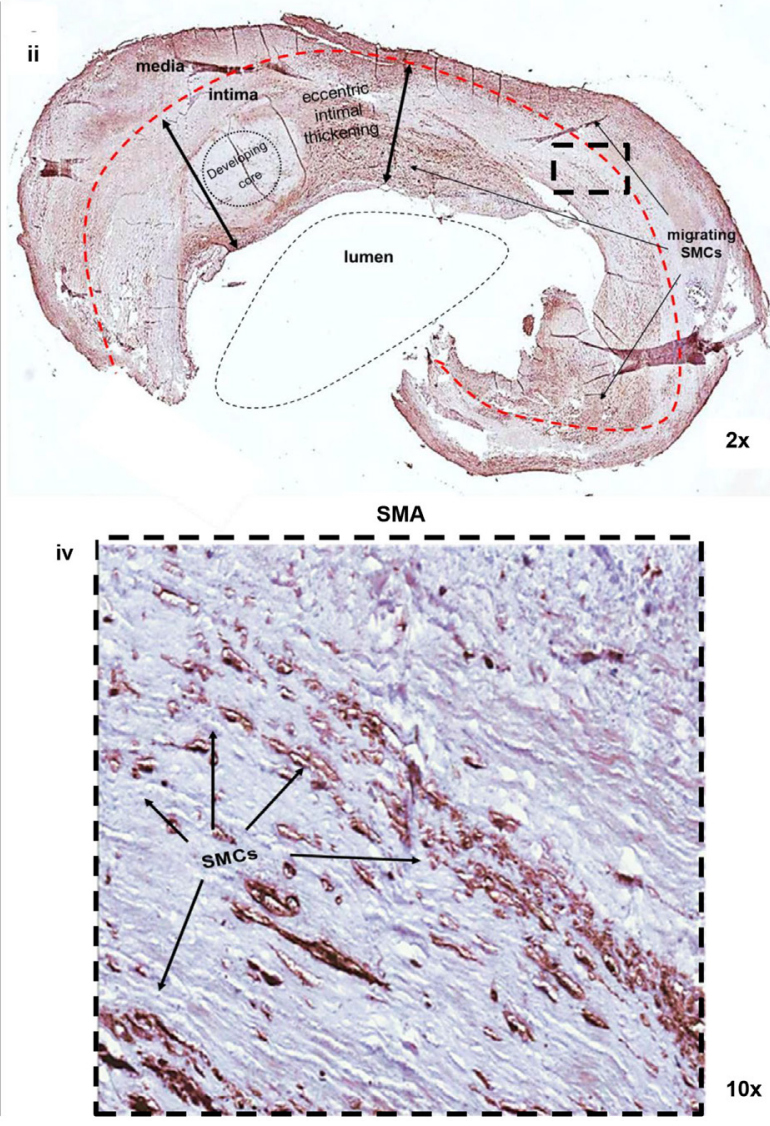



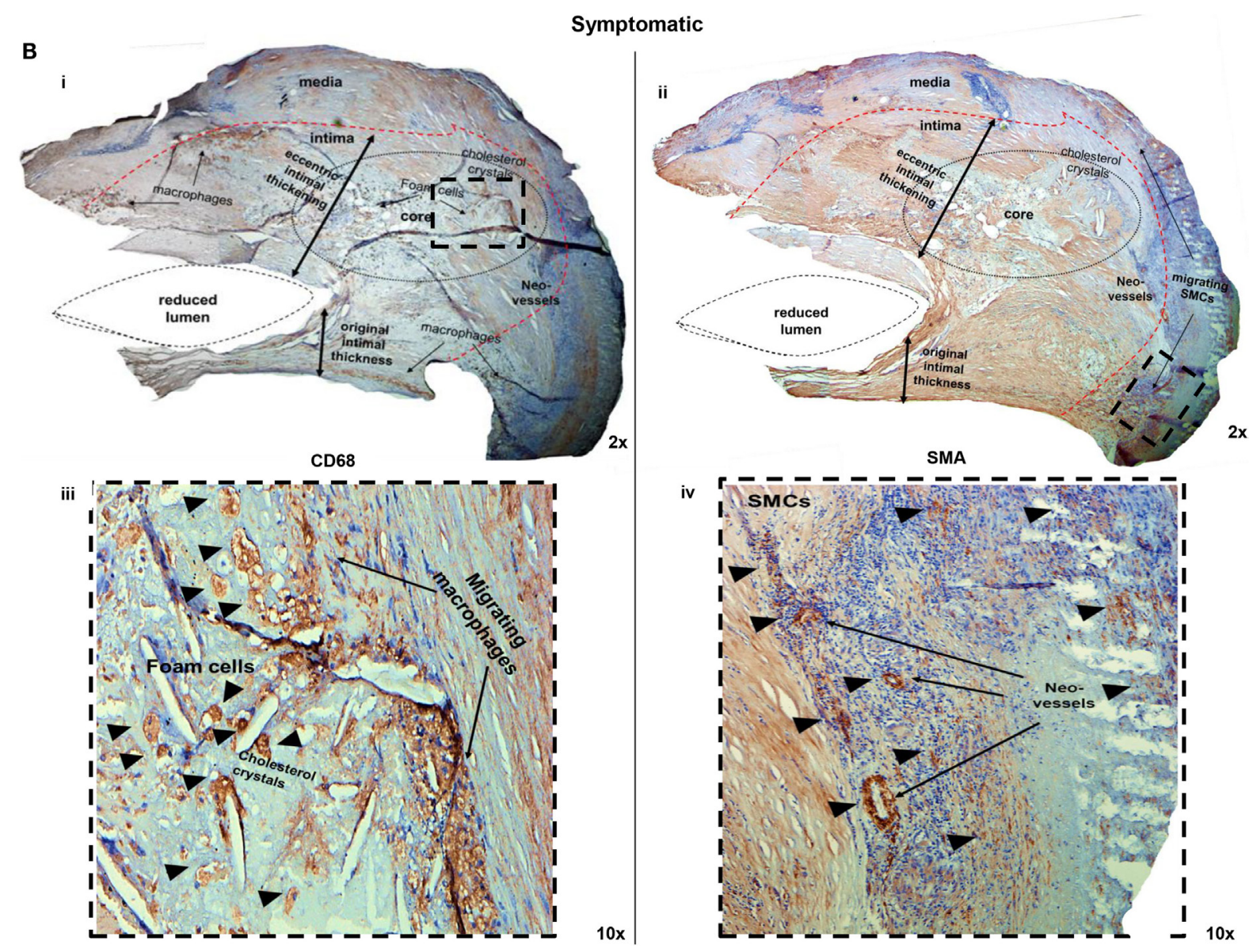

FIGURE 2 | Macrophage/foam cell and SMCs content is decreased in (A) asymptomatic and increased in (B) symptomatic plaques. (A) Low (2x) (i,ii) and high $(10 x)$ (iii,iv) magnifications of the IC portion of a plaque from an asymptomatic patient, where the central lumen, intimal side, and media layer of the plaque are visible. Sections were stained with an anti-CD68 Ab (i,iii) to identify macrophage accumulation. Macrophages were identified migrating toward the developing core with little detection of foam cells (head arrows). Sections were also stained with an anti-SMA Ab (ii,iv). SMCs (arrows) display migratory activity, moving from media to intimal layer. (B) Low (2x) (i,ii) and high (10x) (iii,iv) magnifications, of the IC portion of a plaque from a symptomatic patient, identifying a reduced lumen compared to asymptomatic plaques (i), an intimal side and a media layer of the plaque. The intimal thickness is also increased compared with asymptomatic plaques (i,iii). Macrophages (arrows), detected with an anti-CD68 Ab, are evident in the necrotic core and foam cell accumulation (head arrows) are predominant. (ii,iv) Extensive SMCs activity (head arrows), detected with an anti-SMA Ab, is evident primarily in the intima layer. Pictures were captured using TLM (2x and 10x magnifications). Representative images of $N=3$ independent staining are displayed here.

patients showed greater hemorrhagic activity. Overall, IC was considered the most diseased portion on the basis of the predominant prevalence of calcifications, fibrotic, and necrotic tissue and hemorrhagic events, therefore, it was used in subsequent immunohistochemistry analysis for comparisons with $\mathrm{RDF}$ and for comparison between symptomatic vs. asymptomatic lesions (Figure 1D).

There were obvious differences between the developing core and the necrotic core in asymptomatic and symptomatic plaques. Indeed, early to intermediate stages of the disease are characterized by the fibrotic tissue that starts to replace cells, such as macrophages, foam cells, and SMCs, whereas at later stages, the first visible clinical sign of the disease, known as "fatty-streak" is identified by cholesterol crystal deposits that are highly concentrated in that region (Figure S2 in Supplementary Material). Cholesterol precipitates in the extra cellular matrix as a consequence of the release on the outer space of the lipid content of dead foam cells due to necrotic processes.

\section{Cellular Composition of Symptomatic and Asymptomatic Plaques}

$\mathrm{H} \& \mathrm{E}$ staining was employed to highlight the general structure of the lesions (hemorrhage, calcification, connective tissue, and fibrous/lipid burden). However, identification and localization of specific cell types, such as macrophages, macrophage-derived foam cells, and SMCs, are best achieved using immunohistochemistry analysis, as shown in Figure 2. Combined analysis of the two staining procedures allows to definitively establishing the orientation of the plaque, identifying the luminal side and the intimal and medial layers. Macrophages and foam cells were identified using a human anti-CD68 Ab, as a specific marker (Figure 2A, i,iii and Figure 2B, i,iii). Macrophages were found throughout 
A

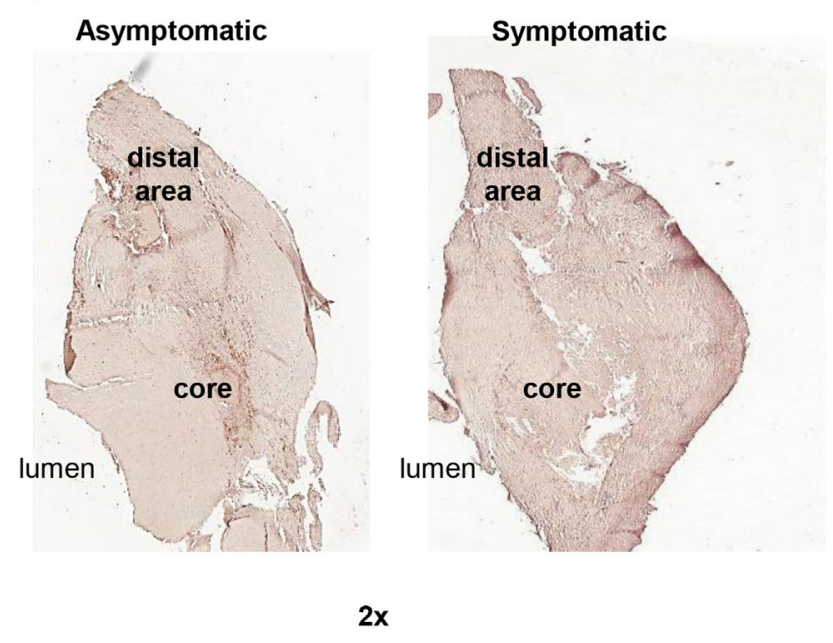

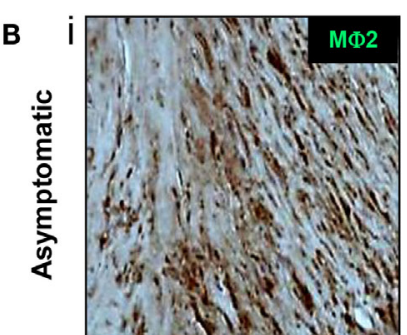
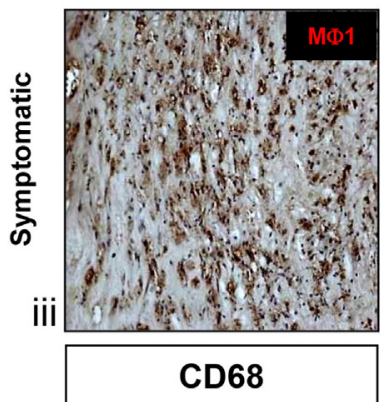

C
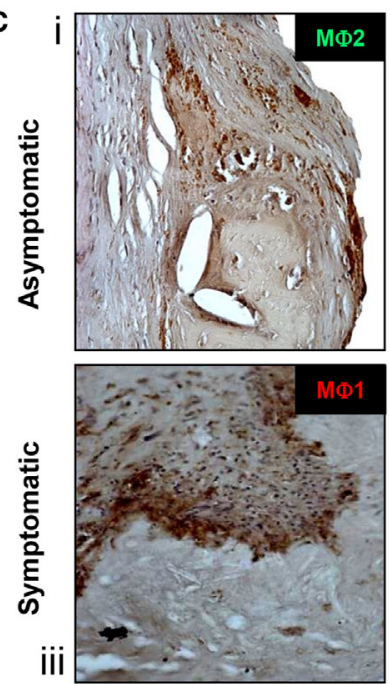
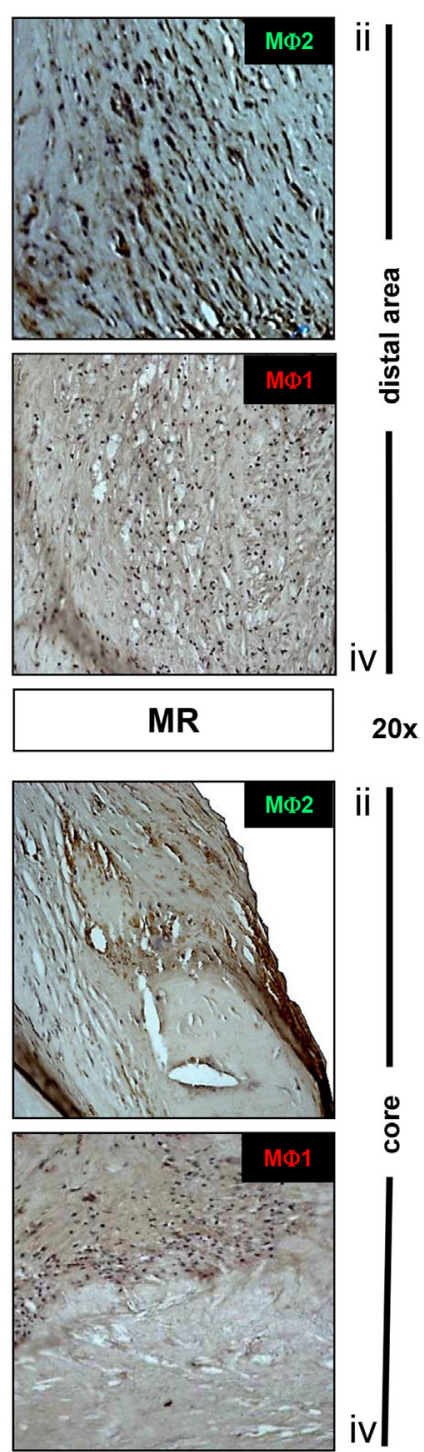

20x

FIGURE 3 | Anti-inflammatory MФ2 macrophages are decreased in symptomatic plaques. (A) In both asymptomatic and symptomatic lesions, a central area, corresponding to the lesion initiation site (core) and a peripheral region (distal area) of the plaque were identified. In the "distal area" of asymptomatic patients, $\mathrm{M \Phi 2}\left(\mathrm{CD}^{+} 8^{+} / \mathrm{MR}^{+}\right)$are predominant (B) (i,ii). Although still present in peripheral areas, MФ2 populations are also located in the developing core of the asymptomatic plaques (C) (i,ii). Conversely, symptomatic lesions are characterized by the predominance of M 1 1 macrophages (CD68 $\left.{ }^{+} / \mathrm{MR}^{-}\right)$, in both the distal and developed core (B) (iii,iv) and (C) (iii,iv). Pictures were captured using TLM (2 and 20x magnifications). Representative images of $N=3$ independent staining are shown.

the plaque sections, while foam cells preferentially localized in the developing or necrotic core of both asymptomatic and symptomatic plaques. In addition, vascular SMCs, determined at $\mathrm{H} \& \mathrm{E}$ level by their characteristic elongated nucleus, were confirmed by IHC using a human anti-SMA Ab, as a specific marker. These cells localize to the medial side of the plaque (Figure 2A, ii,iv and Figure 2B, ii,iv).

In particular, as disease progress, the lumen of the vessel is reduced and the intimal thickness is increased, according to the necrotic core development. Macrophages migrate toward the core, phagocytizing lipids and transforming into foam cells, reaching the forming atheroma, where necrotic processes occur. Here, necrotic foam cells are recognized by the presence of cholesterol crystals, a hallmark of the lipids released in the extra cellular matrix, promoting an inflammatory status (Figure 2B, iii). In symptomatic lesion, extensive SMCs migratory activity is also displayed, moving from media to intima layer, forming the structure of neo-vessels during neo-angiogenetic processes (Figure 2B, iv). Overall, IHC analysis highlights that both cell types are active within atherosclerosis.

\section{Localization and Quantification of MФ1 and MФ2 Macrophage in Lesions}

For the next set of experiments, we focused on the analysis of macrophage subpopulations, the "classically activated" $\mathrm{M} \Phi 1$ pro-inflammatory macrophages and the "alternative" MФ2 
anti-inflammatory macrophage to gain insight into macrophage plasticity during atherosclerotic disease progression. Their localization, differentiation, and distribution relative to one another were initially examined by IHC staining for both CD68, a macrophage marker previously used to identify macrophages in human atherosclerotic plaques $(23,25,42)$, which we used to identify the total macrophage population in each plaque, and for MR, specifically used to discriminate between MФ1 (CD68 ${ }^{+}$/ $\left.\mathrm{MR}^{-}\right)$and $\mathrm{M} \Phi 2\left(\mathrm{CD}^{+} / \mathrm{MR}^{+}\right)$macrophage subpopulations (24). First, a peripheral region, named "distal area," characterized by the original thickness of the vessel prior to the development of the lesion, was identified. Subsequently, a developing or established necrotic region (core) was found in an area characterized by eccentric thickening of the vessel (Figure 3A). IHC analysis of asymptomatic plaques identified that macrophages were only found abundantly in the lipid core of the plaque and rarely in the intimal space (Figure 3B, i,ii and Figure 3C, i,ii). By contrast, in symptomatic plaques, macrophage accumulation was evident in both the developed lipid core and the distal area of the diseased portion of the plaque (Figure 3B, iii,iv and Figure 3C, iii,iv). Our data based on the expression of $\mathrm{MR}$, relative to the presence of CD68 marker, concurs with that of others, show that there is more than one population of macrophage present in atherosclerotic lesions. Furthermore, we showed that $\mathrm{MR}^{+}$macrophages in asymptomatic plaques were localized to more stable distal areas and were also modestly present around the developing core, compared to MФ1 macrophages; while in symptomatic plaques, there was a decrease in MR expression specifically in the core but also to some extent in the distal area, as evidenced in macrophage localization analysis of symptomatic plaques. The specificity of this staining was verified by the use of negative controls (Figure S1 in Supplementary Material).

\section{Differential Expression of $\mathrm{M \Phi 1}$ and $\mathbf{M \Phi 2}$ Macrophage Markers in Symptomatic and Asymptomatic Atherosclerotic Patients}

Several markers predominantly expressed by specific subsets of macrophages have now been identified (43). The aim of the next series of experiments was to quantify differential expression of established MФ1/MФ2 markers and Th1/Th2 cytokines and chemokines in human atherosclerotic plaque and to address if there are changes in gene and/or protein expression associated with disease progression.

In addition, due to the fact that a major function of macrophages in the context of atherosclerotic disease progression is in cholesterol trafficking, we examined the expression of the SR SRA-1 and the ABC-transporter proteins ABCA1 and ABCG1 to identify if they are associated with either an $\mathrm{M} \Phi 1$ or $\mathrm{M} \Phi 2$ phenotype and if they are altered in human disease progression.

As shown in Figure 4, CD36 is almost equally expressed in asymptomatic and symptomatic plaque. Similarly, the expression of CD14 was unaltered between asymptomatic and symptomatic plaques. However, the previously described pan-macrophage marker CD68 mRNA level was increased by 7.3 -fold $(p<0.05)$ in symptomatic relative to asymptomatic plaques, suggesting that the total number of macrophages is higher in symptomatic lesions.

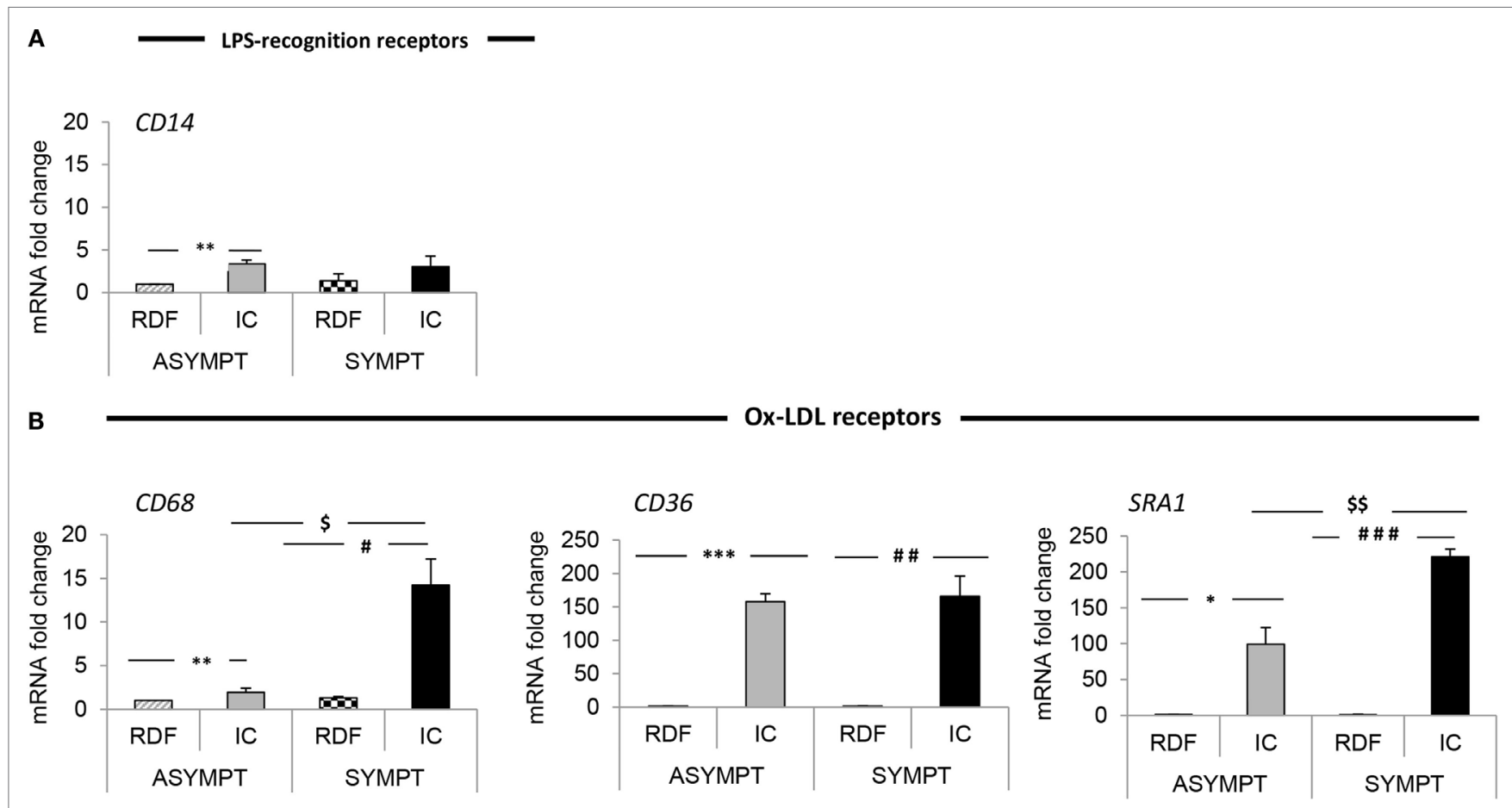

FIGURE 4 | Continued 


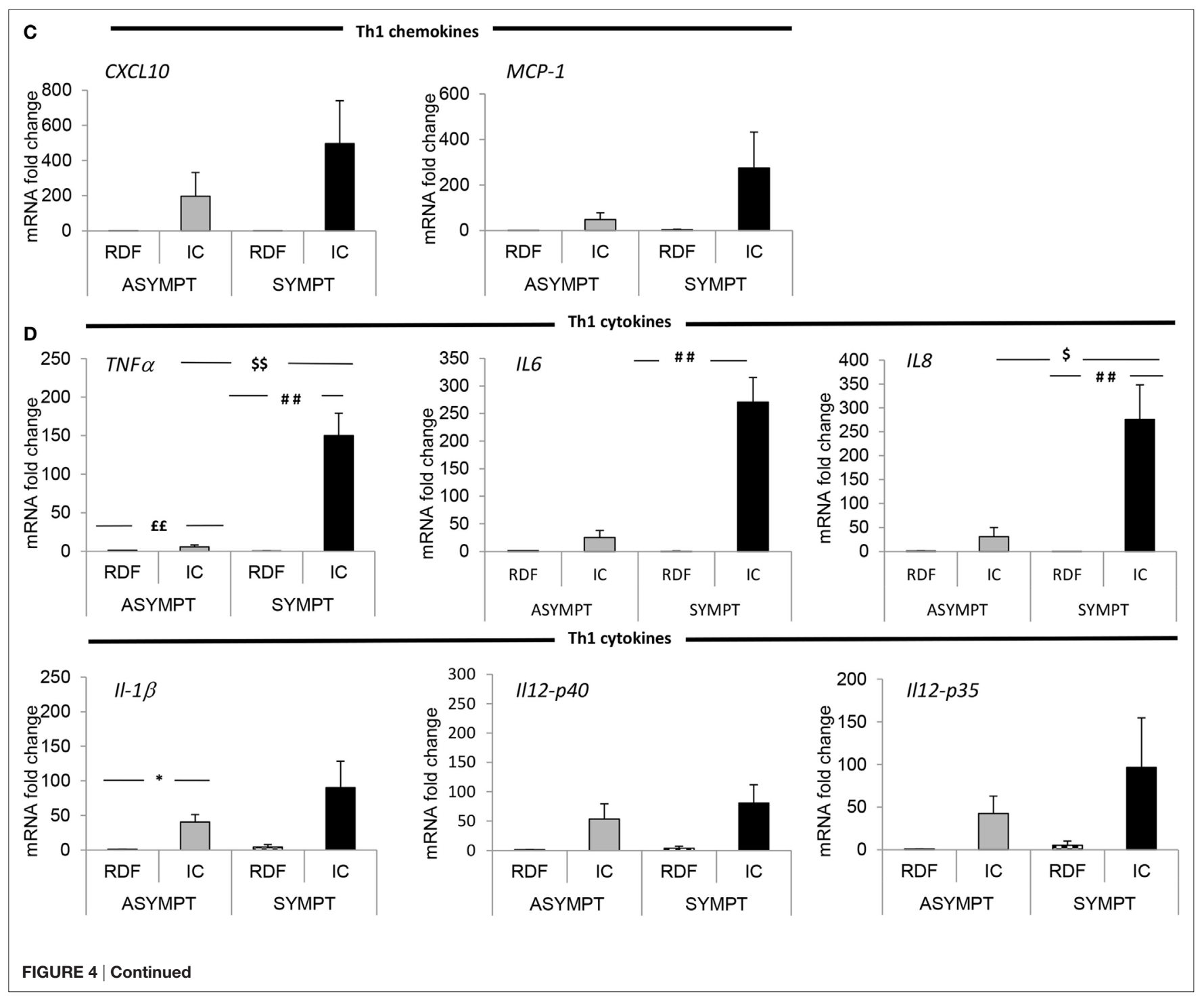

Indeed, the change in CD68 expression is in keeping with the increased expression of $M \Phi 1$ markers and Th1 cytokines namely, TNF $\alpha$, IL-6, and IL- 8 all of which were increased in the IC of symptomatic plaques compared with asymptomatic (by 26.6-fold $p<0.01$; 10.8 -fold, $p<0.01$; and 8.9 -fold, $p<0.05$, respectively) (Table 1). In addition, there was a trend toward increased expression of CXCL10, MCP-1, and IL-1 $\beta$ expression, although this did not reach significance (Table 3; Figure 4). These data, together with the increase in CD68 expression, suggest that the total number of macrophages is higher in symptomatic plaques and is mainly composed of $\mathrm{M} \Phi 1$.

Conversely, MФ2 markers, MR, CD163, and Dectin-1 mRNA levels were decreased in symptomatic compared to asymptomatic (by 3.7-fold, $p<0.001$; 3.5-fold, $p<0.05$, and 3.20, $p<0.05$, respectively). Furthermore, the expression of Th2 cytokines IL-10 and IL-13 and Th2 chemokine CCL22 were significantly decreased in symptomatic compared with asymptomatic plaques (by 3.2-fold, $p<0.05$; 8.9-fold, $p<0.05$, and 4.8 -fold, $p<0.01$, respectively) and again there was a trend toward decreased
IL-4 and CCL18 expression (Table 3; Figure 4), suggesting a decrease in MФ2 macrophages in the progression of human atherosclerosis.

Interestingly, expression of the SR SRA-1 and the efflux proteins, ABCA1 and ABCG1, were also increased in symptomatic compared to asymptomatic (by 2.2-fold, $p<0.01 ; 2.8$-fold, $p<0.01$; and 4.2-fold, $p<0.05$, respectively). This suggests that the increase in SR and ABC- transporter expression is associated with an $M \Phi 1-$ like phenotype. In summary, the total number of macrophages is higher in symptomatic lesions, which are mainly composed of $\mathrm{M} \Phi 1$ and displaying a high expression of Th1 cytokines and SRA-1, ABCA1, and ABCG1 expression. Conversely, although fewer in absolute number of macrophages, asymptomatic lesions are mainly composed of MФ2 macrophage, characterized by an up-regulation of MR, CD163, and Dectin-1 and Th2 cytokines and chemokines.

Western blot analysis of representative macrophage markers altered between asymptomatic and symptomatic patients, namely CD68, MR, and cholesterol efflux ABCA1, confirmed 


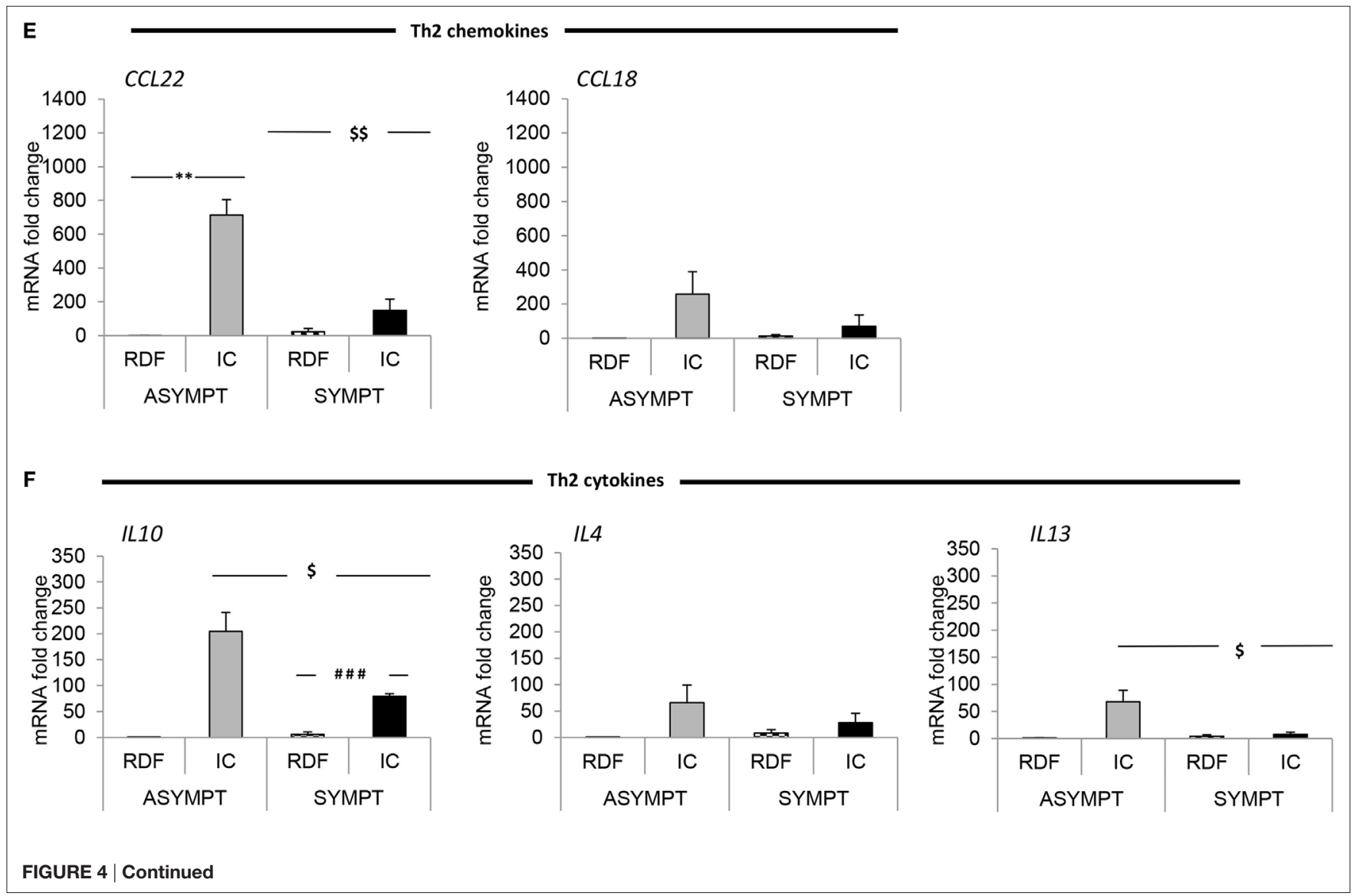

the mRNA expression data. Briefly, CD68 expression in asymptomatic plaques was decreased by 3 -fold $(p<0.001)$ compared to symptomatic plaques; MR expression was increased by 2 -fold $(p<0.05)$ and ABCA1 was decreased by 2.7 -fold $(p<0.05)$ (Figures 5A,B).

To further expand protein analysis, quantification of IHC stained section was used to identify macrophage subpopulations. Using the "Nuclear" and the "Color Deconvolution" algorithms CD68 and MR protein expression was quantified (Figure 5C). The data clearly illustrate that the number of total macrophage in asymptomatic patients is significantly lower than those of symptomatic patients [represented by only $57 \pm 12 \%$ of the total number of macrophage in the symptomatic group $(p<0.05)]$ (Figure 5C - bars). Although MФ2 macrophages are relatively abundant in asymptomatic plaques, representing $42 \pm 5 \%$ of the total macrophage population, this population significantly decreases with progression of disease, where MФ2 macrophages represent only the $23 \pm 3 \%$ of the total macrophage population in symptomatic plaques $(p<0.05)$. Furthermore, the relative percentage of $M \Phi 1$ is significantly higher in symptomatic plaques, compared to asymptomatic plaques where this population represents $77 \pm 5 \%$ vs. $58 \pm 2 \%$ of the total macrophage population in symptomatic and asymptomatic plaques, respectively (Figure 5C). The change in relative abundance of $M \Phi 2$ populations as disease progresses is likely due to both an increase in the number of pro-inflammatory macrophages and a direct effect on the anti-inflammatory fraction. These data, based on localization and quantification of MФ1 and MФ2 macrophage subpopulations, within both symptomatic and asymptomatic plaques suggest that the MФ2 macrophage subpopulation may play potential protective role in human atherosclerosis. However, detailed characterization of the macrophage populations warrant further investigation.

\section{DISCUSSION}

Despite atherosclerosis being defined as a chronic inflammatory condition, paired with lipid accumulation, there are no therapies that induce regression of pre-established disease. This is important as most patients present clinically with established disease and the therapeutic goal is to reverse the lesion together with the prevention of the risks associated with the plaque presence, such as plaque rupture and thrombosis. Surgical revascularization is the current intervention employed to treat advanced atherosclerosis. Although clinically endarterectomy is effective, it does not fully address the long-term management of the disease. Therefore, plaque regression is the quintessential target of atherosclerosis intervention. By decreasing plaque size, stenosis can be relieved, removing not only the risks associated with plaque size, rupture, and thrombosis but also reducing the requirement for surgical intervention. There is a general consensus that a stenosis of $<70 \%$ does not require surgical intervention, 
G

Hb-Hp scavenger receptor

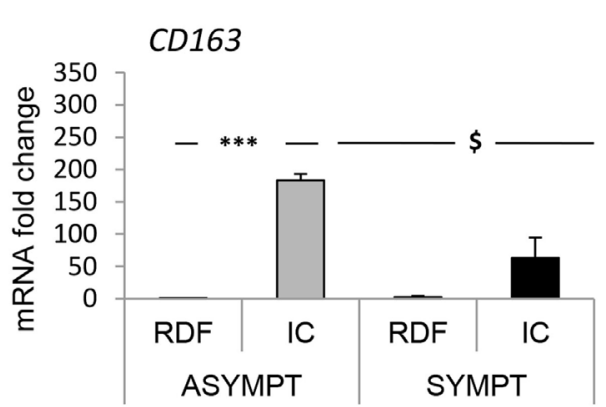

H

Polysaccharide receptors

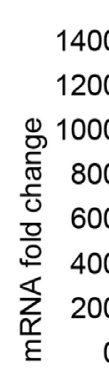

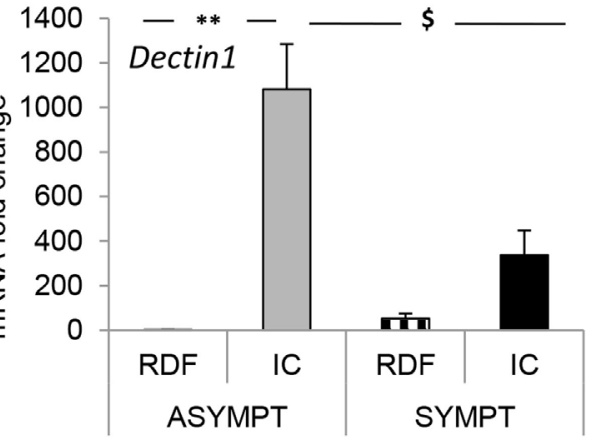

I

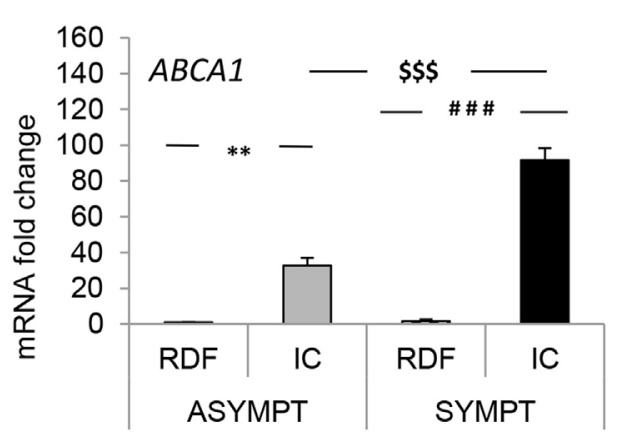

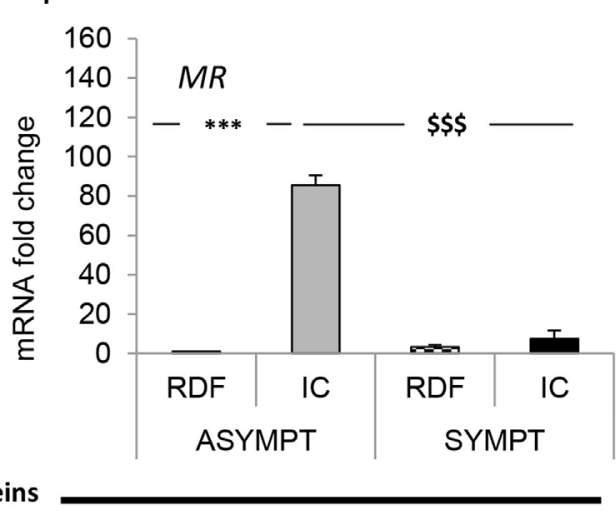

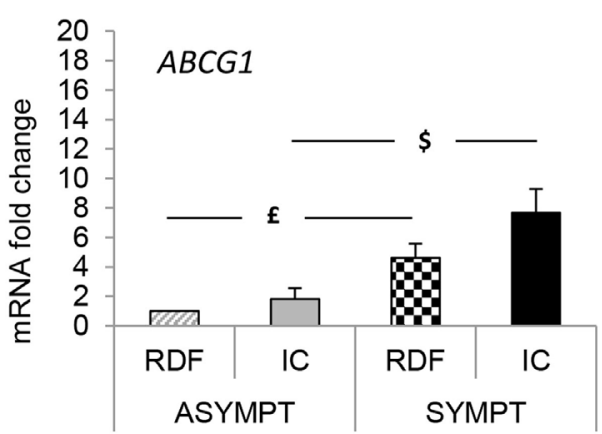

FIGURE 4 | Macrophage polarization marker mRNA analysis in symptomatic vs. asymptomatic patients. The panels display mRNA fold change expression of 22 macrophage markers over control (RDF in asymptomatic patients). RT-PCR analysis showed that (A) in both asymptomatic and symptomatic plaques low level of the LPS-recognition receptor, CD14 is expressed. In symptomatic plaques the macrophage profile is characterized by increased expression of (B) ox-LDL scavenger receptors (CD68, CD36, and SRA-1), Th1-associated (C) chemokines (CXCL10 and MCP-1) and (D) cytokines (TNF $\alpha$, IL-1 $\beta$, IL-6, IL-8, $\mathrm{IL}-12 \mathrm{p} 40$, and IL-12p35) and (I) efflux proteins (ABCA1 and ABCG1), compared with asymptomatic patients, suggesting a predominant M $\Phi$ 1 phenotype among the whole macrophage population. Conversely, asymptomatic lesions are primarily composed of M $\Phi 2$-like macrophages, characterized by increased expression of Th2-associated (E) chemokines (CCL22 and CCL18) and (F) cytokines (IL-10, IL-4, and IL-13), (G) Hb-Hp scavenger receptor (CD163) and (H)

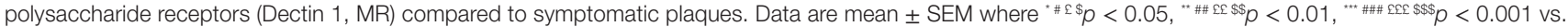
controls, after $T$-test analysis of $N=3$ independent experiments. Controls legend: * indicates comparison between RDF vs. IC in asymptomatic; \# indicates comparison between RDF vs. IC in symptomatic; ${ }^{\varepsilon}$ indicates comparison between RDFs in the two subgroups of patients; ${ }^{\$}$ indicates comparison between ICs in the two subgroups of patients.

as the risks of surgery outweigh the risks presented by stenosis. However, stenosis $>70 \%$ is deemed to be a greater risk than its surgical removal. Regression of an atherosclerotic lesion into the " $<70 \%$ " stenosis group in itself would be therapeutically beneficial $(9,44)$.

Our laboratory has previously shown that dietary administration of CLA induces regression of pre-established atherosclerosis in vivo (16) and has identified the monocyte/macrophage as the cellular target through which CLA mediates this profound effect $(17,18)$. Importantly, we have previously shown that, in a CLA-induced model of atherosclerosis regression, there is enrichment of genes associated with the MФ2 alternatively activated macrophage phenotype in the murine aorta (19). This is in agreement with other published data that show decreased lipid 
TABLE 3 | Differential gene expression for established macrophage markers in symptomatic vs. asymptomatic human carotid plaques.

\begin{tabular}{|c|c|c|c|c|c|c|c|}
\hline \multicolumn{2}{|l|}{ Gene } & \multicolumn{2}{|c|}{ Asymptomatic } & \multicolumn{2}{|c|}{ Symptomatic } & \multicolumn{2}{|c|}{ IC Sympt vs. IC Asympt } \\
\hline Function & Name & RDF & IC & RDF & IC & Fold change & $p$-Value \\
\hline LPS-recognition receptor & CD14 & 1 & 3.36 & 1.39 & 3.06 & 0.91 & 0.8309 \\
\hline \multirow[t]{3}{*}{ Ox-LDL scavenger receptors } & $C D 68$ & 1 & 1.94 & 1.29 & 14.23 & 7.32 & 0.0152 \\
\hline & CD36 & 1 & 157.99 & 1.01 & 165.85 & 1.05 & 0.8197 \\
\hline & SRA-1 & 1 & 99.34 & 0.76 & 221.13 & 2.23 & 0.0087 \\
\hline \multirow[t]{2}{*}{ Th1 chemokines } & CXCL10 & 1 & 196.41 & 0.99 & 496.03 & 2.53 & 0.3428 \\
\hline & MCP-1 & 1 & 48.34 & 3.37 & 274.51 & 5.68 & 0.2319 \\
\hline \multirow[t]{6}{*}{ Th1 cytokines } & TNF $\alpha$ & 1 & 5.64 & 0.18 & 150.16 & 26.64 & 0.0077 \\
\hline & $\| \mathrm{L}-1 \beta$ & 1 & 40.58 & 4.27 & 90.40 & 2.23 & 0.0877 \\
\hline & IL-6 & 1 & 25.13 & 0.42 & 270.79 & 10.78 & 0.0060 \\
\hline & IL-8 & 1 & 31.06 & 0.29 & 276.31 & 8.90 & 0.0297 \\
\hline & IL-12p40 & 1 & 53.34 & 3.70 & 80.72 & 1.51 & 0.5378 \\
\hline & IL-12p35 & 1 & 42.55 & 5.26 & 96.80 & 2.28 & 0.4272 \\
\hline \multirow[t]{2}{*}{ Th2 chemokine } & CCL22 & 1 & 713.12 & 22.66 & 148.34 & -4.81 & 0.0076 \\
\hline & CCL18 & 1 & 258.03 & 12.72 & 70.63 & -3.65 & 0.2731 \\
\hline \multirow[t]{3}{*}{ Th2 cytokines } & $I L-10$ & 1 & 204.66 & 5.79 & 79.08 & -3.15 & 0.0273 \\
\hline & IL-4 & 1 & 65.72 & 8.41 & 28.32 & -2.32 & 0.3812 \\
\hline & $I L-13$ & 1 & 67.88 & 3.78 & 7.65 & -8.87 & 0.0484 \\
\hline \multirow[t]{2}{*}{ Polysaccharide receptors } & Dectin-1 & 1 & 1081.59 & 51.50 & 337.56 & -3.20 & 0.0320 \\
\hline & MR & 1 & 85.56 & 3.21 & 7.46 & -3.74 & 0.0003 \\
\hline \multirow[t]{2}{*}{ Efflux proteins } & $A B C A 1$ & 1 & 32.66 & 1.64 & 91.72 & 2.81 & 0.0019 \\
\hline & ABCG1 & 1 & 1.81 & 4.61 & 7.68 & 4.23 & 0.0290 \\
\hline Hb-Hp scavenger receptor & $C D 163$ & 1 & 182.92 & 2.73 & 63.03 & -3.54 & 0.0222 \\
\hline
\end{tabular}

mRNA fold change expression of 22 macrophage markers over control (RDF in asymptomatic patients) analyzed by RT-PCR. A fold change analysis of ICs between the two groups is also reported, together with the relative $p$-value after T-test correction of $N=3$ independent experiments. A value of $p<0.05$ was considered significant. Genes which were significantly altered are indicated in bold.

levels are accompanied by lower plaque lipid content and higher expression of genes for MФ2 macrophages in the Reversa mouse model of regression (36).

Most recently, we have shown that CLA primes human peripheral blood monocytes to adapt an $M \Phi 2$ phenotype in vitro thus mediating atheroprotection, suggesting that interventional tools capable of altering the macrophage infiltrate toward an anti-inflammatory phenotype may be of therapeutic relevance (20). However, to translate this into a clinical setting, a comprehensive understanding of macrophage populations in human atherosclerotic disease progression is required. Indeed, to date, studies on macrophage populations and polarization in human atherosclerotic disease progression are limited.

To address these points, a study was designed in which atherosclerotic plaques from asymptomatic and symptomatic patients undergoing CEA were recruited. To confirm if this was a robust model of disease progression, initial experiments addressed if it was possible to identify cellular changes between the two patient subgroups and, ultimately, if differential macrophage target gene regulation could be detected.

To establish if the differences between symptomatic and asymptomatic disease could be seen at cytological level, the cellular content of atherosclerotic lesions was first verified using H\&E. This provided a useful tool in the identification of lesion composition and orientation, highlighting the differences "between" and "within" lesion types. Indeed, the differences in cellular composition were evident upon gross analysis of the plaques and it was observed that asymptomatic plaques are generally less diseased and present with less evidence of hemorrhaging and stenosis. The characteristic differences seen at a cellular level between symptomatic and asymptomatic plaques were also evident, with increased intimal thickening, cholesterol crystal deposition, foam cells, and lipid accumulation and a larger and more developed lipid core seen in the symptomatic plaque compared to the asymptomatic plaque. Moreover, the results presented here show less cellular activity in the asymptomatic compared with symptomatic plaques. $\mathrm{RDF}$ was deemed to an appropriate control for the purpose of these studies. It was used as an internal control within the plaque, displaying minimal lipid deposition, SMCs and fibrous deposits, thus indicating a "relative" absence of atherosclerotic development.

As described above, the strategy provides a good model of disease progression and, therefore, this study was extended to identify macrophage subpopulations in asymptomatic and symptomatic plaques, initially using an immunohistochemical approach.

A major aim was to identify the presence of $M \Phi 2$ antiinflammatory macrophages, and to seek evidence that there is a shift in $M \Phi 2$ population associated with disease progression. We have recently shown in vitro that increasing MФ2 subpopulations plays a role in atheroprotection (20). Immunohistochemistry analysis was performed using the CD68 pan-macrophage marker, which identified the total number of macrophages present. MФ2 macrophages were then discriminated by the staining of $\mathrm{MR}$ receptor. It was predicted that $M \Phi 2$ staining would be scarce in the symptomatic plaques compared to the asymptomatic plaques. The analysis showed that MФ2 anti-inflammatory macrophages were mainly located in more distal regions and were found overlying the developing 


\section{protein}

A

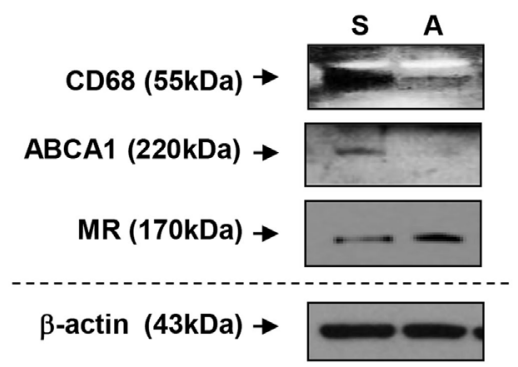

C

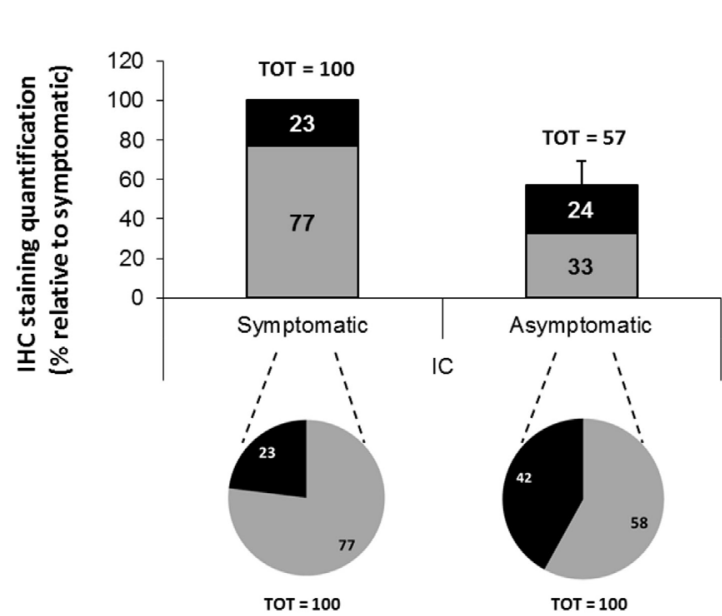

B

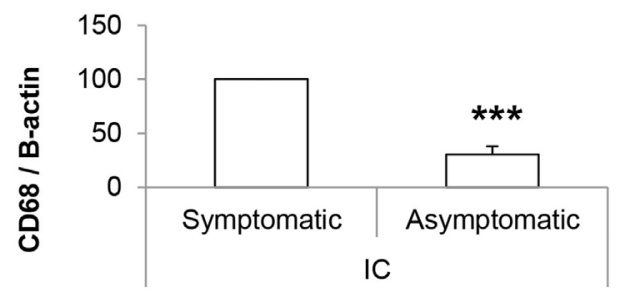

-M2 = MR $\square M 1=$ CD68-MR
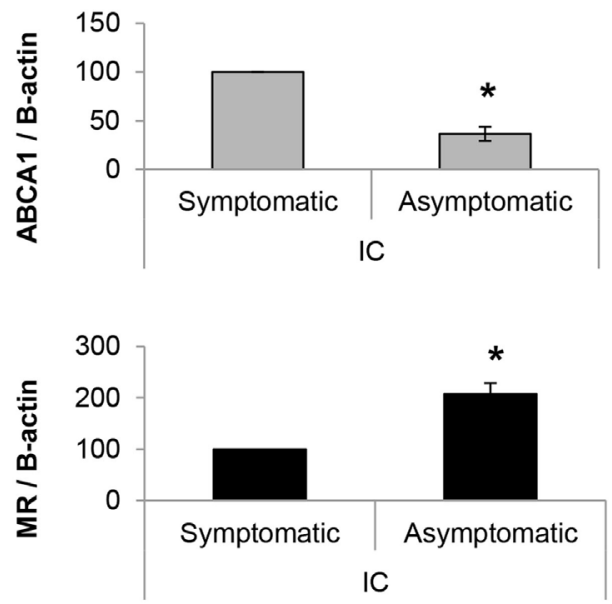

FIGURE 5 | Analysis of protein expression of MФ1 and MФ2 markers in symptomatic vs. asymptomatic patients. (A) Western Blot and (B) the relative densitometry analysis, confirm RT-PCR analysis of selected macrophage markers, where ABCA1 is increased and MR is decreased in symptomatic (S) compared with asymptomatic (A) plaques, suggesting a switch from $\mathrm{M} \Phi 2$ to $M \Phi 1$ macrophage thus enriching the pro-inflammatory fraction population, as disease progresses.

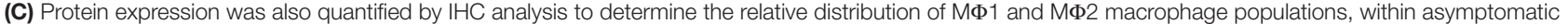
and symptomatic plaques. Quantification of CD68 and MR staining is shown. In symptomatic plaques, M $\Phi 1$ macrophages are increased relative to M $\Phi 2$. In

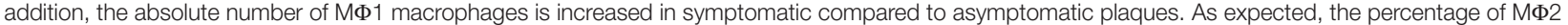
macrophage, relative to $\mathrm{M} \Phi 1$ cells, is significantly greater in asymptomatic compared to symptomatic patients (pie charts), even though their absolute number between symptomatic and symptomatic lesions is similar (bars). For all the experiments above mentioned, data are mean \pm SEM where ${ }^{\star} p<0.05$, ${ }^{* *} p<0.01$, ${ }^{\star * *} p<0.001$ vs. symptomatic after $T$-test analysis of $N=3$ independent experiments.

core in the asymptomatic plaques. It was noted that, as the disease progressed, these MФ2 alternative macrophages were suppressed and MФ1 pro-inflammatory macrophages became predominant features of the plaque. M $\Phi 1$ classically activated macrophages were present in abundance in the developed lipid core of the symptomatic plaque and were rarely found in the intimal regions of the plaque. МФ2 levels were evidently higher in asymptomatic atherosclerotic plaques, which are ultimately less advanced in their disease progression. Furthermore, in line with our findings, Bouhlel et al. showed that both $M \Phi 1$ and MФ2 macrophages have been identified in human atherosclerotic plaque and that $\mathrm{M} \Phi 2$ macrophages are present at more stable locations within atherosclerotic plaques (23). However, that study did not compare asymptomatic and symptomatic patients. In addition, a previous study by Lee et al. employed laser micro-dissection to examine macrophage mRNA from stable and ruptured plaques using a transcriptomics approach and found significant differences in the gene expression profiles. Of relevance to our study, where we report increase in cholesterol efflux proteins and inflammatory cytokines in disease progression, they showed that Leptin and FABP4, which both have functions linking lipid metabolism to inflammation, were among the most differentially expressed individual genes in disease progression (10). Furthermore, in a separate study, transcriptomics and immunohistochemistry was employed to analyze macrophage subset dynamics in successive stages of atherosclerosis. In keeping with our data, they also showed

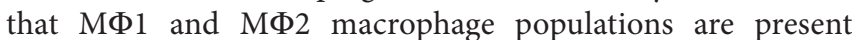


throughout disease development and that $M \Phi 1$ macrophages are preferentially linked to plaque progression (42).

In this study, we further extended our immunohistochemistry approach to perform nuclear analysis, used to quantify the number of CD68 and MR expressing cells. This novel strategy used an algorithm, "V9 Aperio Nuclear Algorithm," to determine levels of a target protein in human atherosclerotic plaques, providing an accurate and compelling result. This tool allowed quantification of the percentage of $М \Phi 2$ macrophage relative to $M \Phi 1$ macrophage in both asymptomatic and symptomatic plaques. "Aperio" digital scanning and quantification is a novel methodology and, for the first time, these types of algorithms have been used to quantify macrophage subsets in human atherosclerotic tissue.

As expected, the proportion of $М \Phi 2$ macrophages relative to MФ1 was much greater in the asymptomatic plaques' developing core compared to symptomatic. Interestingly, based on unique localization and differential expression of MR, substantial evidence confirmed the presence of at least two macrophage subpopulations within atherosclerotic lesions, as well as strong indication that there is a relative loss of $\mathrm{M} \Phi 2$ and a proportional increase in $M \Phi 1$ macrophages in symptomatic atherosclerotic lesion development.

Finally, characterization of ABCA1 and MR expression confirmed the presence of $M \Phi 1$ and $M \Phi 2$ subpopulations within lesions, at both mRNA and protein levels. Specifically, MФ1 were characterized by high levels of Th1 cytokines and chemokines, ox-LDL SRs and efflux proteins and low levels of Th2 cytokines and chemokines, polysaccharide receptors and $\mathrm{Hb}-\mathrm{Hp} \mathrm{SR}$, while the inverse was defined for $M \Phi 2$. Our novel data on expression of $M \Phi 1$ and $M \Phi 2$ markers in human plaques are summarized in Table 4. It has been suggested that there may be an additional "resident macrophage" subpopulation present alongside MФ1 and MФ2 macrophages (45) and in future studies, a specific marker for this unknown population should be used to determine its specific localization and role in atherosclerotic progression. It is important to note that this study employed analysis of whole plaque specimens. To definitively characterize the macrophage response in atherosclerotic disease progression isolation of macrophages from harvested samples followed by identification of their transcriptome and proteome should be performed in a larger population.

Macrophages have previously been characterized as heterogeneous cell populations $(23,24)$. In work by ChinettiGbaguidi et al. and Bouhlel et al., it has been proposed that PPAR $\gamma$ activation may effectively prime the differentiation of primary human macrophage into $М Ф 2$ phenotype, generating anti-inflammatory and atheroprotective populations. More recently, it has been shown that $M \Phi 1$ macrophage content of atherosclerotic plaques is associated with clinical incidence of ischemic stroke and increased inflammation or fibrinolysis (32). In the context of atherosclerosis, it has been shown that there is an $M \Phi 2$ to $M \Phi 1$ switch during plaque progression, likely due to a conversion of cells already present in the lesion, suggesting that interventional tools, able to revert the macrophage infiltrate toward the MФ2 phenotype, may exert an atheroprotective action (32).
TABLE 4 | Macrophage profiling in asymptomatic and symptomatic atherosclerotic patients.

\begin{tabular}{|c|c|c|c|c|}
\hline & \multicolumn{4}{|c|}{ Macrophage Profiling } \\
\hline & \multicolumn{2}{|l|}{ Gene } & \multicolumn{2}{|c|}{ Level of expression } \\
\hline & $\begin{array}{l}\text { Function } \\
\text { LPS-recognition } \\
\text { receptor }\end{array}$ & $\begin{array}{l}\text { Name } \\
\text { CD14 }\end{array}$ & $\begin{array}{c}\text { Asymptomatic } \\
\text { Low }\end{array}$ & $\begin{array}{c}\text { Symptomatic } \\
\text { Low }\end{array}$ \\
\hline \multirow[t]{4}{*}{$\begin{array}{l}\text { MФ1-like } \\
\text { phenotype }\end{array}$} & $\begin{array}{l}\text { Ox-LDL } \\
\text { scavenger } \\
\text { receptors }\end{array}$ & $\begin{array}{l}\text { CD68 } \\
\text { CD36 } \\
\text { SRA-1 }\end{array}$ & Low & High \\
\hline & Efflux proteins & $\begin{array}{l}\text { ABCA1 } \\
\text { ABCG1 }\end{array}$ & Low & High \\
\hline & Th1 chemokines & $\begin{array}{l}\text { CXCL10 } \\
\text { MCP-1 }\end{array}$ & Low & High \\
\hline & Th1 cytokines & $\begin{array}{l}\text { TNF } \alpha \\
\text { IL-1 } \beta \\
\text { IL-6 } \\
\text { IL-8 } \\
\text { IL-12p40 } \\
\text { IL-12p35 }\end{array}$ & Low & High \\
\hline \multirow[t]{4}{*}{$\begin{array}{l}\text { МФ2-like } \\
\text { phenotype }\end{array}$} & Th2 chemokine & $\begin{array}{l}\text { CCL22 } \\
\text { CCL18 }\end{array}$ & High & Low \\
\hline & Th2 cytokines & $\begin{array}{l}\| \mathrm{L}-10 \\
\mathrm{IL}-4 \\
\mathrm{IL}-13\end{array}$ & High & Low \\
\hline & $\begin{array}{l}\text { Polysaccharide } \\
\text { receptors }\end{array}$ & $\begin{array}{l}\text { Dectin-1 } \\
\text { MR }\end{array}$ & High & Low \\
\hline & $\begin{array}{l}\mathrm{Hb}-\mathrm{Hp} \text { scavenger } \\
\text { receptor }\end{array}$ & CD163 & High & Low \\
\hline
\end{tabular}

Summary of macrophage profiling based on differential mRNA differential expression of the 22 macrophage markers between symptomatic and asymptomatic atherosclerotic plaques. In asymptomatic patients, the macrophage displays an MФ2-like phenotype, displaying high levels of Th2 cytokines and chemokines, polysaccharide receptors and the Hb-Hp scavenger receptor. Conversely, in symptomatic patients, macrophages display an MФ1-like phenotype, characterized by high levels of Th1 cytokines and chemokines, ox-LDL scavenger receptors, and efflux proteins, associated with atherosclerotic disease progression.

We have shown that alteration in macrophage populations in human disease drives atherosclerosis progression. Therefore, future work, aimed to better understand the mechanism through which $M \Phi 1-M \Phi 2$ shift is induced needs to be performed. The ultimate aim of this investigatory field is to find novel therapeutic targets in the treatment of human atherosclerosis, as an alternative type of intervention to the surgical, procedure. Therefore, the experimental model described above will also facilitate identification of shift in macrophage populations in response to current and novel therapeutics.

\section{AUTHOR CONTRIBUTIONS}

MdG participated in the design of the study, carried out the experiments and prepared the manuscript. DC participated in the design of the study and facilitated analysis. $\mathrm{MB}$ performed surgical carotid endarterectomy in patients. $\mathrm{OB}$ conceived and 
designed the study and prepared the manuscript. All authors read and approved the final manuscript.

\section{ACKNOWLEDGMENTS}

This work was supported by grants TRA/2007/04 from the Health Research Board (Ireland). The authors acknowledge the support

\section{REFERENCES}

1. Nathan L, Chaudhuri G. Estrogens and atherosclerosis. Annu Rev Pharmacol Toxicol (1997) 37:477-515. doi:10.1146/annurev.pharmtox.37.1.477

2. Fabryova L, Cagan S. [Relation between insulin resistance and small, dense lipoproteins with low density and the development of atherosclerosis in type 2 diabetes mellitus]. Bratisl Lek Listy (1998) 99(3-4):138-45.

3. Mehta JL, Saldeen TG, Rand K. Interactive role of infection, inflammation and traditional risk factors in atherosclerosis and coronary artery disease. J Am Coll Cardiol (1998) 31(6):1217-25. doi:10.1016/S0735-1097(98)81787-7

4. Handschin C, Spiegelman BM. The role of exercise and PGClalpha in inflammation and chronic disease. Nature (2008) 454(7203):463-9. doi:10.1038/ nature 07206

5. Moore KJ, Tabas I. Macrophages in the pathogenesis of atherosclerosis. Cell (2011) 145(3):341-55. doi:10.1016/j.cell.2011.04.005

6. Viiri LE, Full LE, et al. Smooth muscle cells in human atherosclerosis: proteomic profiling reveals differences in expression of Annexin A1 and mitochondrial proteins in carotid disease. J Mol Cell Cardiol (2013) 54:65-72. doi:10.1016/j.yjmcc.2012.11.002

7. Moreno PR, Falk E, Palacios IF, Newell JB, Fuster V, Fallon JT. Macrophage infiltration in acute coronary syndromes. Implications for plaque rupture. Circulation (1994) 90(2):775-8. doi:10.1161/01.CIR.90.1.94

8. Hopkins LN, White CJ, Foster MT, Powell RJ, Zemel G, Diaz-Cartelle J. Carotid artery stenting and patient outcomes: the CABANA surveillance study. Catheter Cardiovasc Interv (2014) 84(6):997-1004. doi:10.1002/ccd. 25578

9. Fuster V, Badimon JJ, Chesebro JH. Atherothrombosis: mechanisms and clinical therapeutic approaches. Vasc Med (1998) 3(3):231-9. doi:10.1177/ 1358836X9800300310

10. Robbins CS, Hilgendorf I, Weber GF, Theurl I, Iwamoto Y, Figueiredo JL, et al. Local proliferation dominates lesional macrophage accumulation in atherosclerosis. Nat Med (2013) 19(9):1166-72. doi:10.1038/nm.3161

11. Lusis AJ. Atherosclerosis. Nature (2000) 407(6801):233-41. doi:10.1038/ 35025203

12. Chawla A, Boisvert WA, Lee CH, Laffitte BA, Barak Y, Joseph SB, et al. A PPAR gamma-LXR-ABCA1 pathway in macrophages is involved in cholesterol efflux and atherogenesis. Mol Cell (2001) 7(1):161-71. doi:10.1016/ S1097-2765(01)00164-2

13. Tall AR, Costet P, Wang N. Regulation and mechanisms of macrophage cholesterol efflux. J Clin Invest (2002) 110(7):899-904. doi:10.1172/JCI0216391

14. Panzenboeck U, Kratzer I, Sovic A, Wintersperger A, Bernhart E, Hammer A, et al. Regulatory effects of synthetic liver $\mathrm{X}$ receptor- and peroxisomeproliferator activated receptor agonists on sterol transport pathways in polarized cerebrovascular endothelial cells. Int J Biochem Cell Biol (2006) 38(8):1314-29. doi:10.1016/j.biocel.2006.01.013

15. Banni S. Conjugated linoleic acid metabolism. Curr Opin Lipidol (2002) 13(3):261-6. doi:10.1097/00041433-200206000-00005

16. Toomey S, Harhen B, Roche HM, Fitzgerald D, Belton O. Profound resolution of early atherosclerosis with conjugated linoleic acid. Atherosclerosis (2006) 187(1):40-9. doi:10.1016/j.atherosclerosis.2005.08.024

17. McClelland S, Cox C, O'Connor R, de Gaetano M, McCarthy C, Cryan L, et al. Conjugated linoleic acid suppresses the migratory and inflammatory phenotype of the monocyte/macrophage cell. Atherosclerosis (2010) 211(1): 96-102. doi:10.1016/j.atherosclerosis.2010.02.003 of the UCD Conway Institute Transcriptomics and Imaging Core Facilities.

\section{SUPPLEMENTARY MATERIAL}

The Supplementary Material for this article can be found online at http://journal.frontiersin.org/article/10.3389/fimmu.2016.00275

18. de Gaetano M, Dempsey E, Marcone S, James WG, Belton O. Conjugated linoleic acid targets beta2 integrin expression to suppress monocyte adhesion. J Immunol (2013) 191(8):4326-36. doi:10.4049/jimmunol.1300990

19. McCarthy C, Duffy MM, et al. IL-10 mediates the immunoregulatory response in conjugated linoleic acid-induced regression of atherosclerosis. FASEB J (2013) 27(2):499-510. doi:10.1096/fj.12-215442

20. de Gaetano M, Alghamdi K, Marcone S, Belton O. Conjugated linoleic acid induces an atheroprotective macrophage MPhi2 phenotype and limits foam cell formation. J Inflamm (Lond) (2015) 12:15. doi:10.1186/s12950-015-0060-9

21. Chinetti-Gbaguidi G, Staels B. Macrophage polarization in metabolic disorders: functions and regulation. Curr Opin Lipidol (2011) 22(5):365-72. doi:10.1097/MOL.0b013e32834a77b4

22. Shalhoub J, Falck-Hansen MA, Davies AH, Monaco C. Innate immunity and monocyte-macrophage activation in atherosclerosis. J Inflamm (Lond) (2011) 8:9. doi:10.1186/1476-9255-8-9

23. Bouhlel MA, Derudas B, Rigamonti E, Dièvart R, Brozek J, Haulon S, et al. PPARgamma activation primes human monocytes into alternative M2 macrophages with anti-inflammatory properties. Cell Metab (2007) 6(2):137-43. doi:10.1016/j.cmet.2007.06.010

24. Chinetti-Gbaguidi G, Baron M, Bouhlel MA, Vanhoutte J, Copin C, Sebti Y, et al. Human atherosclerotic plaque alternative macrophages display low cholesterol handling but high phagocytosis because of distinct activities of the PPARgamma and LXRalpha pathways. Circ Res (2011) 108(8):985-95. doi:10.1161/CIRCRESAHA.110.233775

25. Cojocaru E, Trandafirescu M, Leon M, Cotuţiu C, Foia L. Immunohistochemical expression of anti-CD68 antibody in atherosclerotic plaque. Rom J Morphol Embryol (2012) 53(1):61-6.

26. Waldo SW, Li Y, Buono C, Zhao B, Billings EM, Chang J, et al. Heterogeneity of human macrophages in culture and in atherosclerotic plaques. Am J Pathol (2008) 172(4):1112-26. doi:10.2353/ajpath.2008.070513

27. Shaikh S, Brittenden J, Lahiri R, Brown PA, Thies F, Wilson HM. Macrophage subtypes in symptomatic carotid artery and femoral artery plaques. Eur J Vasc Endovasc Surg (2012) 44(5):491-7. doi:10.1016/j.ejvs.2012.08.005

28. Cho KY, Miyoshi H, Kuroda S, Yasuda H, Kamiyama K, Nakagawara J, et al. The phenotype of infiltrating macrophages influences arteriosclerotic plaque vulnerability in the carotid artery. J Stroke Cerebrovasc Dis (2013) 22(7):910-8. doi:10.1016/j.jstrokecerebrovasdis.2012.11.020

29. Kunjathoor VV, Febbraio M, Podrez EA, Moore KJ, Andersson L, Koehn S, et al. Scavenger receptors class A-I/II and CD36 are the principal receptors responsible for the uptake of modified low density lipoprotein leading to lipid loading in macrophages. J Biol Chem (2002) 277(51):49982-8. doi:10.1074/ jbc.M209649200

30. Boytard L, Spear R, Chinetti-Gbaguidi G, Acosta-Martin AE, Vanhoutte J, Lamblin N, et al. Role of proinflammatory CD68(+) mannose receptor(-) macrophages in peroxiredoxin-1 expression and in abdominal aortic aneurysms in humans. Arterioscler Thromb Vasc Biol (2013) 33(2):431-8. doi:10.1161/ATVBAHA.112.300663

31. Pilling D, Fan T, Huang D, Kaul B, Gomer RH. Identification of markers that distinguish monocyte-derived fibrocytes from monocytes, macrophages, and fibroblasts. PLoS One (2009) 4(10):e7475. doi:10.1371/journal.pone.0007475

32. Chistiakov DA, Bobryshev YV, Nikiforov NG, Elizova NV, Sobenin IA, Orekhov AN. Macrophage phenotypic plasticity in atherosclerosis: the associated features and the peculiarities of the expression of inflammatory genes. Int J Cardiol (2015) 184:436-45. doi:10.1016/j.ijcard.2015.03.055 
33. Taylor ME, Bezouska K, Drickamer K. Contribution to ligand binding by multiple carbohydrate-recognition domains in the macrophage mannose receptor. J Biol Chem (1992) 267(3):1719-26.

34. Feinberg H, Park-Snyder S, Kolatkar AR, Heise CT, Taylor ME, Weis WI. Structure of a C-type carbohydrate recognition domain from the macrophage mannose receptor. J Biol Chem (2000) 275(28):21539-48. doi:10.1074/jbc. M002664200

35. Etzerodt A, Moestrup SK. CD163 and inflammation: biological, diagnostic, and therapeutic aspects. Antioxid Redox Signal (2013) 18(17):2352-63. doi:10.1089/ars.2012.4834

36. Elcombe SE, Naqvi S, Van Den Bosch MW, MacKenzie KF, Cianfanelli F, Brown GD, et al. Dectin-1 regulates IL-10 production via a MSK1/2 and CREB dependent pathway and promotes the induction of regulatory macrophage markers. PLoS One (2013) 8(3):e60086. doi:10.1371/journal.pone. 0060086

37. Viola J, Soehnlein O. Atherosclerosis - a matter of unresolved inflammation. Semin Immunol (2015) 27(3):184-93. doi:10.1016/j.smim.2015.03.013

38. Mahmood DF, Abderrazak A, Couchie D, Lunov O, Diderot V, Syrovets T, et al. Truncated thioredoxin (Trx-80) promotes pro-inflammatory macrophages of the M1 phenotype and enhances atherosclerosis. J Cell Physiol (2013) 228(7):1577-83. doi:10.1002/jcp.24319

39. Jessup W, Gelissen IC, Gaus K, Kritharides L. Roles of ATP binding cassette transporters A1 and G1, scavenger receptor BI and membrane lipid domains in cholesterol export from macrophages. Curr Opin Lipidol (2006) 17(3):247-57. doi:10.1097/01.mol.0000226116.35555.eb

40. Grewal T, Priceputu E, Davignon J, Bernier L. Identification of a gamma-interferon-responsive element in the promoter of the human macrophage scavenger receptor A gene. Arterioscler Thromb Vasc Biol (2001) 21(5):825-31. doi:10.1161/01.ATV.21.5.825
41. McCarthy C, Lieggi NT, Barry D, Mooney D, de Gaetano M, James WG, et al. Macrophage PPAR gamma Co-activator-1 alpha participates in repressing foam cell formation and atherosclerosis in response to conjugated linoleic acid. EMBO Mol Med (2013) 5(9):1443-57. doi:10.1002/emmm.201302587

42. Stöger JL, Gijbels MJ, van der Velden S, Manca M, van der Loos CM, Biessen EA, et al. Distribution of macrophage polarization markers in human atherosclerosis. Atherosclerosis (2012) 225(2):461-8. doi:10.1016/j. atherosclerosis.2012.09.013

43. Gomez Perdiguero E, Klapproth K, Schulz C, Busch K, Azzoni E, Crozet L, et al. Tissue-resident macrophages originate from yolk-sac-derived erythromyeloid progenitors. Nature (2015) 518(7540):547-51. doi:10.1038/nature13989

44. Henriksson M, Lundgren F, Carlsson P. Cost-effectiveness of endarterectomy in patients with asymptomatic carotid artery stenosis. Br J Surg (2008) 95(6):714-20. doi:10.1002/bjs.6157

45. Jenkins SJ, Ruckerl D, Cook PC, Jones LH, Finkelman FD, van Rooijen N, et al. Local macrophage proliferation, rather than recruitment from the blood, is a signature of TH2 inflammation. Science (2011) 332(6035):1284-8. doi:10.1126/science.1204351

Conflict of Interest Statement: The authors declare that the research was conducted in the absence of any commercial or financial relationships that could be construed as a potential conflict of interest.

Copyright (C) 2016 de Gaetano, Crean, Barry and Belton. This is an open-access article distributed under the terms of the Creative Commons Attribution License (CC BY). The use, distribution or reproduction in other forums is permitted, provided the original author(s) or licensor are credited and that the original publication in this journal is cited, in accordance with accepted academic practice. No use, distribution or reproduction is permitted which does not comply with these terms. 